\title{
A hybrid model integrating Elman neural network with variational mode decomposition and Box-Cox transformation for monthly runoff time series prediction
}

\author{
Yan Kang ( $\sim$ kangyan@nwsuaf.edu.cn ) \\ Northwest A\&F University: Northwest Agriculture and Forestry University \\ Fangqin Zhang \\ Northwest A\&F University: Northwest Agriculture and Forestry University \\ Xiao Cheng \\ Northwest A\&F University: Northwest Agriculture and Forestry University \\ Peiru Chen \\ Northwest A\&F University: Northwest Agriculture and Forestry University \\ Songbai Song \\ Northwest A\&F University: Northwest Agriculture and Forestry University
}

\section{Research Article}

Keywords: Monthly runoff prediction, non-stationary and skewed runoff time series, Variational mode decomposition, Box-Cox transformation, Elman neural network, Wei River Basin

Posted Date: March 7th, 2022

DOl: https://doi.org/10.21203/rs.3.rs-807243/v1

License: (c) (i) This work is licensed under a Creative Commons Attribution 4.0 International License.

Read Full License 
1 A hybrid model integrating Elman neural network with variational mode decomposition

2 and Box-Cox transformation for monthly runoff time series prediction

3 Fangqin Zhang ${ }^{1,2}$, Yan Kang ${ }^{1,2,}$, , Xiao Cheng1, Peiru Chen ${ }^{1}$, Songbai Song,

$4 \quad{ }^{1}$ College of Water Resources and Architectural Engineering, Northwest A\&F University, Yangling 712100, China.

$5 \quad{ }^{2}$ Key Laboratory of Agricultural Soil and Water Engineering in Arid and Semiarid Areas, Ministry of Education, Northwest A\&F

6 University, Yangling 712100, China.

7 Abstract: Precise and reliable monthly runoff prediction plays a vital role in optimal management of

8 water resources but non-stationarity and skewness of monthly runoff time series can pose major

9 challenges for developing appropriate prediction models. To address these issues, this paper proposes

10 a novel hybrid prediction model based on Elman neural network (Elman), variational mode

11 decomposition (VMD) and Box-Cox transformation (BC), named VMD-BC-Elman model. Firstly, the

12 observed runoff is decomposed into sub-time series using VMD for the better frequency resolution.

13 Secondly, the input datasets were transformed into normal distribution using Box-Cox, and as a result,

14 skewedness in the data was removed and the correlation between the input and output variables

15 enhanced. Finally, Elman is used to simulate the respective sub-time series. The proposed model is

16 evaluated using monthly runoff time series at Zhangjiashan, Zhuangtou and Huaxian hydrological

17 stations in Wei River Basin in China. The model performances are compared with those of single

18 models (SVM, Elman), decomposition-based (VMD-SVM, VMD-Elman et.al) and BC-based models

19 (BC-SVM and BC-Elman) by employing four metrics. The results show that the hybrid models

20 outperform single models, and VMD-BC-Elman model performs best in all considered hybrid models

21 with NSE greater than 0.95 , R greater than 0.98 , NMSE less than $4.73 \%$, and PBIAS less than $0.39 \%$

22 in both training and testing periods. The study indicates that VMD-BC-Elman model is a satisfactory

23 data-driven approach to predict the non-stationary and skewed monthly runoff time series, representing 
24 an effective tool for predicting monthly runoff series.

25 Key words: Monthly runoff prediction; non-stationary and skewed runoff time series; Variational

26 mode decomposition; Box-Cox transformation; Elman neural network; Wei River Basin

\section{1. Introduction}

Accurate and reliable runoff prediction is of great significance for the water resources planning

29 and management, flood controlling and drought warning, which has attracted the extensive attention

30 as a vital and difficult topic in hydrology modeling (Mohamad et al. 2017; Peng et al. 2011). However,

31 runoff time series show complex non-linearity, non-stationarity and skewness (Barge and Sharif 2016),

32 which are attributable to climate changes and frequent human activities, such as the variations of

33 precipitation, conservancy project, urbanization, and so on. As a result, it is a great challenge to

34 accurately simulate the intrinsic dynamic process of the inconsistent runoff series (Feng et al. 2020).

35 In recent years, a multitude of runoff prediction models have been developed to model runoff time

36 series, and representative models generally include two categories: process-driven models and data-

37 driven models. Process-driven models, which are based on strict physical mechanisms, can

38 successfully characterize the rainfall-runoff processes with complex mathematical formula, accurate

39 meteorology and hydrological knowledge (Partington et al. 2012). However, there have been key

40 modeling controversies about the issues such as adequacy of process parameterizations, data

41 limitations and uncertainty, and computational constraints on model analysis (Demirel et al. 2009;

42 Clark et al. 2017), which may lead up to poor performance of process-driven models. In contrast, data-

43 driven approaches, which are based on the hydrological statistics and machine learning, can achieve 
44 satisfactory performance without too much information input. The data-driven model can be

45 categorized by three main methods: statistical methods, artificial intelligent methods (AI) and hybrid

46 models (Myronidis et al. 2018; Kuremoto et al. 2014; Tiwari et al., 2013). Statistical models, such as

47 autoregressive model (AR) (Sarlak 2008), autoregressive moving average model (ARMA) (Moeeni et

48 al. 2017) and so on, have been widely applied to capture the stationary and linear relationship between

49 variables and they, however, are not suitable for modeling non-stationary and non-linear runoff time

50 series. AI models, i.e. neural network models (Sedki et al. 2008), support vector machines (Aggarwal

51 et al. 2012), and random forest (Bojang et al. 2020), have been extensively applied in the hydrology

52 prediction. Compared to the conventional regression models, AI models have greatly improved

53 prediction performance for highly nonlinear runoff time series. As a typical recurrent neural network

54 model, Elman neural network (Elman), equipped with a time delay operator, has short-term memory

55 ability and is very suitable for the time series forecasting, and parameter selection is simple (Krishnan

56 et al. 2019). Support vector machine (SVM), based on Vapnik-Chervonenkis dimension and structural

57 risk minimization, can map data from input space to high-dimensional space in terms of a kernel

58 function selected to suit the problem, and attain the optimal solution for regression issues (Karamouz

59 et al. 2009). However, standalone AI models have a limited ability to identify the intrinsic non-

60 stationary features of the input data, resulting in problems that the Elman model is inclined to fall into

61 local optimum, over-fitting for, and the SVM model strongly dependents on the parameters selection

62 (Wang et al. 2013). Therefore, a hybrid model which combines AI methods with data processing

63 techniques has become the most popular option for predicting non-stationary runoff time series (Wen

64 et al. 2019). 
In recent years, signal decomposition algorithms, used as data processing techniques, are widely

66 developed to effectively extract the multiple frequency information hidden from the complex runoff

67 series, and generalizability of the data-driven model can be improved (Zhang et al. 2015). Empirical

68 mode decomposition (EMD) and ensemble empirical mode decomposition (EEMD) are commonly

69 employed as time-frequency decomposition algorithms. Among them, EMD has a strong adaptability

70 due to a self-adaptive data-driven tool, but it lacks bases for rigorous mathematical theories and is

71 prone to edge effect and ensuing modal aliasing (Huang et al. 2003; Sankaran and Reddy 2016). As an

72 improved model of EMD, EEMD algorithm is proposed by adding white noise into a series of intrinsic

73 mode functions (IMFs) which eliminates aliasing of modes effectively (Wu and Huang 2009). However,

74 the amplitude of the added white noise in EEMD model is determined by expert experience, and the

75 modal components cannot be controlled adaptively, which maybe lead to loss of information or

76 incomplete decomposition (Yu et al. 2018). To address these issues, Variational Mode Decomposition

77 (VMD), which has a sound mathematical basis, strong anti-aliasing ability and good decomposition

78 potential, is introduced to recursively decompose original non-stationary series into a group of

79 relatively stable sub-series (Drcgomiretskiy 2014). As a new signal decomposition method, VMD has

80 been applied to speech recognition, fault analysis and hydrology forecasting in recent years, and the

81 models have shown a satisfactory performance for signal decomposition (Deb et al. 2020; Mohanty et

82 al. 2018; Li et al. 2020), and the hybrid model of VMD and support vector machine (SVM) has

83 achieved good forecasting results (Feng et al. 2020).

84 The above hybrid models can process the non-stationary characteristics of runoff time series using

85 decomposition, but the influence of runoff skewness on the forecasting performance has been rarely 
86 explored by researchers. Normalization transformation techniques of data processing are worthy of

87 more attention. Box-Cox transformation can remove skewness of runoff data and transform the data

88 into more normal distribution which significantly stabilize variance of the runoff time series, and

89 improve the normality and linearity of data series, reduce the probability of pseudo regression, and

90 effectively improve the correlation between the input and output variables. The Box-Cox has achieved

91 good normality in hydrology frequency analysis and calculation (Vasiliades et al. 2009; Seong 2014),

92 but it has been rarely employed in runoff prediction models.

93 In summary, the AI models, especially the Elman neural network, has rarely integrated with VMD

94 and Box-Cox methods for runoff prediction. Therefore, to further improve the prediction accuracy, a

95 novel hybrid VMD-BC-Elman model is proposed for monthly runoff prediction in this paper The

96 objectives of this study are as followed: (1) VMD is used to decompose the original non-stationary

97 monthly runoff time series into several relatively stationary sub-time series; (2) the Box-Cox

98 transformation is employed to normalize the candidate input variables for each sub-time series; (3) a

99 hybrid model incorporating VMD, Box-Cox, and Elman is constructed, and tested using runoff data

100 collected from Wei River Basin; (4) the robustness and generalizability of the proposed hybrid model 101 is evaluated by comparing it to other combinations of models.

\section{2. Methodologies}

\section{$103 \quad 2.1$ Variational Mode Decomposition}

104 Variational mode decomposition (VMD), a novel non-recursive signal processing algorithm 105 proposed by Dragomiretskiy and Zosso, is utilized to adaptively decompose a complex non-stationary 106 signal into a set of discrete bandwidth-limited modes in a spectral domain by Wienner filering 
107 (Dragomiretskiy and Zosso 2014). Given the original runoff time series $f(t)(t=1,2, . ., n)$, regarded as

108 a non-stationary signal, it is decomposed into $K$ different modal functions $\mu_{k}(t)(k=1,2, . ., K)$ with

109 central frequency $\omega_{k}$. The bandwidth and central frequency of each mode can be estimated by seeking

110 the optimums of constrained variational modes. The objective function is that the sum of the estimated

111 bandwidths of all sub-series is minimized, while the constraint satisfies that the sum of each

112 decomposed mode is equal to the original signal, which can be expressed as:

113

$$
\begin{aligned}
& \min _{\left\{\mu_{k}\right\},\left\{\omega_{k}\right\}}\left\{\sum_{k=1}^{K}\left\|\partial_{t}\left[\left(\delta(t)+\frac{j}{\pi t}\right) \otimes \mu_{k}(t)\right] e^{-j \omega_{k} t}\right\|_{2}^{2}\right\} \\
& \text { s.t. } \sum_{k=1}^{K} \mu_{k}=f(t)
\end{aligned}
$$

114 where $\left\{\mu_{k}\right\}$ is the set of modes, $\left\{\omega_{k}\right\}$ is the set of central frequencies of modes, $\delta_{t}$ represents the

115 Dirac distribution, $K$ is the number of modes, and $\otimes$ denotes a convolution operation.

The above constrained problem can be transformed to an unconstrained variational problem by

117 introducing Lagrange multiplier and quadratic penalty to construct the augmented Lagrange term L:

$$
\begin{gathered}
L\left(\left\{\mu_{\mathrm{k}}\right\},\left\{\omega_{k}\right\}, \lambda\right)=\alpha \sum_{k=1}^{K}\left\|\partial(t)\left[\left(\delta(t)+\frac{j}{\pi t}\right) \otimes \mu_{k}(t)\right] e^{-j \omega_{k} t}\right\|_{2}^{2}+ \\
\left\|f(t)-\sum_{k=1}^{K} \mu_{k}(t)\right\|_{2}^{2}+\left\langle\lambda(t) f(t)-\sum_{k=1}^{K} \mu_{k}(t)\right\rangle
\end{gathered}
$$

119 Where $\alpha$ and $\lambda$ denote the penalty parameter and Lagrange multiplier, respectively.

120 The alternating direction method of multipliers (ADMM), an effective splitting method for

121 separable optimization problems (Feng et al. 2020), is employed to seek out the saddle points of the

122 augmented Lagrange term, and, therefore, the optimums of the decision variables $\mu_{k}$ and $\omega_{k}$ are 123 presented as follows: 


$$
\left\{\begin{array}{c}
\hat{\mu}_{\mathrm{k}}^{n+1}(\omega)=\frac{\hat{f}(\omega)-\sum_{i \neq k} \hat{\mu}_{i}^{n}(\omega)+\frac{\hat{\lambda}^{n}(\omega)}{2}}{1+2 \alpha\left(\omega-\omega_{n}^{k}\right)^{2}} \\
\omega_{k}^{n+1}=\frac{\int_{0}^{\infty} \omega\left|\hat{\mu}_{k}^{n+1}(\omega)\right|^{2} d \omega}{\int_{0}^{\infty}\left|\hat{\mu}_{k}^{n+1}(\omega)\right|^{2} d \omega} \\
\hat{\lambda}^{n+1}(\omega)=\hat{\lambda}^{n}(\omega)+\tau\left(\hat{f}(\omega)-\sum_{k} \mu_{k}^{n+1}(\omega)\right)
\end{array}\right.
$$

125 where $n$ is the number of iterations, $\tau$ is the update parameters of Lagrange multipliers, and $\hat{\mu}_{k}^{n+1}(\omega)$, $\hat{f}(\omega), \hat{\mu}_{i}^{n}(\omega)$, and $\hat{\lambda}^{n+1}(\omega)$ represent the fast Fourier transform of $\mu_{k}^{n+1}(t), f(t), \mu_{i}^{n}(t)$, and $\lambda^{n}(t)$, respectively.

\subsection{Box-Cox transformation}

The Box-Cox power transformation, proposed by Box and Cox in 1964, is applied to stabilize

variance and remove skewness of time series, and convert them into more normal like distribution

131 (Box and Cox 1964; Hamasaki and $\operatorname{Kim} 2008)$. Supposing $y_{i}(i=1,2, \ldots n)$ is a positive random variable, the Box-Cox transformation with parameter $\lambda$ is defined as:

$$
y_{i}^{(\lambda)}=\left\{\begin{array}{l}
\frac{y_{i}^{\lambda}-1}{\lambda}, \lambda \neq 0 \\
\log y_{i}, \lambda=0
\end{array}, i=1,2, \ldots n\right.
$$

134 where $y_{i}$ is the original signal $\left(y_{i}>0\right), y_{i}^{(\lambda)}$ is the transformed signal, and $\lambda$ denotes the transform 135 coefficient.

136 In order to estimate the parameters of the model, the transformed maximum likelihood function 137 is adopted as the following (Hamasaki and Kim 2008),

$$
\max (L)=-\frac{n}{2} \ln \sigma^{2}+(\lambda-1) \sum_{i=1}^{n} \ln y_{i}
$$

where $L$ denotes the likelihood function, $\sigma^{2}$ is the variance of $y_{i}^{(\lambda)}$. 
141 formula (5).

\section{$142 \quad$ 2.3 Elman Neural Network}

143 The Elman neural network, first proposed by Elman in 1990, is a dynamic recurrent neural 144 network with feedforward connections (Chandra 2015). The Elman network consists of four layers: 145 input layer, hidden layer, context layer, and output layer.

146 Compared with feedforward neural networks, the additional context layer in the Elman neural 147 network is employed to record the output information of the previous moment from the hidden layer 148 and relay the former state in the current iteration, so the Elman neural network is sensitive to the 149 historical input information and more suitable for time series forecasting (Mehrgini et al. 2019). Since 150 the runoff time series have short-term dependency, the Elman neural network is suitable for runoff 151 forecasting. The architecture is shown in Fig. 1.

Fig. 1

The mathematical form can be expressed as (Li et al. 2019):

$$
O_{t}=g\left(\omega_{3} H_{t}+b_{1}\right)
$$

$$
H_{t}=f\left(\omega_{1} C_{t}+\omega_{2} I_{t}+b_{2}\right)
$$

$$
C_{t}=H_{t-1}
$$

157 where $I_{t}, H_{t}, C_{t}$ and $O(t)$ denote input, hidden, context and output layer vector, respectively. $\omega_{1}, \omega_{2}$

158 and $\omega_{3}$ are weights connecting other two adjacent layers. $g$ and $f$ are the activation functions of the

159 hidden layer and the output layer, respectively. $b_{1}$ and $b_{2}$ represent the bias vector. 
According to the above-mentioned methods, the hybrid model is proposed and diagrammed in

162 Fig.2. The modeling procedures are described as follows:

Step1: The original runoff data is decomposed into $k$ sub-time series that are bandwidth-limited

with a center frequency using VMD.

Step2: Each sub-time series from the step 1 is reconstructed using phase space reconstruction

(PSR) to generate candidate input variables series for predicting models, and each reconstructed candidate input variable series is normalized by Box-Cox.

Step3: For each candidate input variables series transformed by Box-Cox from the step 2, LASSO

is employed to rank and select the optimal number of the input variables for Elman model.

Step4: Each sub-time series decomposed by VMD is divided into training dataset and testing

dataset. The parameters can be calibrated by inputting the selected input variables into the Elman, and

172 then the trained model is tested using the testing dataset.

Step5: The final estimates of runoff time series are obtained by summing up the sub-time series

estimated from the predicting models, respectively.

\section{$176 \quad 2.5$ Model performance evaluation}

Nash-Suttcliffe efficiency coefficient (NSE) (Nash and Sutcliffe 1970), the coefficient of

correlation (R), the normalized mean square error (NMSE) (Himanshu et al. 2017), and the percent of 
performance of different models. These metrics are defined as:

$$
\begin{array}{r}
N S E=1-\frac{\sum_{t=1}^{N}\left(Q_{\mathrm{o}}(t)-Q_{\mathrm{p}}(t)\right)^{2}}{\sum_{t=1}^{N}\left(Q_{\mathrm{o}}(t)-\overline{Q_{\mathrm{o}}}\right)^{2}} \\
R=\frac{\sum_{t=1}^{N}\left(Q_{\mathrm{o}}(t)-\overline{Q_{\mathrm{o}}}\right)\left(Q_{\mathrm{p}}(t)-\overline{Q_{\mathrm{p}}}\right)}{\sqrt{\sum_{t=1}^{N}\left(Q_{\mathrm{o}}(t)-\overline{Q_{\mathrm{o}}}\right)^{2}\left(Q_{\mathrm{p}}(t)-\overline{Q_{\mathrm{p}}}\right)^{2}}} \\
N M S E=\frac{\sum_{t=1}^{N}\left(Q_{\mathrm{o}}(t)-Q_{\mathrm{p}}(t)\right)^{2}}{\sum_{t=1}^{N}\left(Q_{\mathrm{o}}(t)\right)^{2}} \times 100 \% \\
P B I A S=\frac{\sum_{t=1}^{N}\left(Q_{\mathrm{o}}(t)-Q_{\mathrm{p}}(t)\right)}{\sum_{t=1}^{N} Q_{\mathrm{o}}(t)} \times 100 \%
\end{array}
$$

185 Where $Q_{\mathrm{o}}(t)$ and $Q_{\mathrm{p}}(t)$ is the observed and predicted runoff, respectively, $\overline{Q_{0}}$ and $\overline{Q_{\mathrm{p}}}$ denotes the 186 mean of observed and predicted monthly runoff time series, respectively. $N$ is the number of $Q_{\mathrm{p}}(t)$.

\section{Case study}

\section{$188 \quad 3.1$ study area and dataset}

The Wei River originates from Weiyuan County in Gansu Province, extends more than $800 \mathrm{~km}$

190 and finally converges into Yellow River (Jiang et al. 2019). The Wei River Basin (WRB) covers an 191 area of approximately $13.5 \times 10^{4} \mathrm{~km}^{2}$ between $104^{\circ} 00^{\prime}-110^{\circ} 20^{\prime} \mathrm{E}$ and $33^{\circ} 50^{\prime}-37^{\circ} 18{ }^{\prime} \mathrm{N}$. Topographically, 192 the elevation of the WRB ranges from $336 \mathrm{~m}$ to $3929 \mathrm{~m}$ (Wang et al. 2020), and a digital elevation 193 model (DEM) of the WRB is presented in Fig.3. The basin is located in arid and semi-arid regions in 194 China, affected by a continental monsoon, and the climate is warm and rainy in summer and cold and 
195 dry in winter, and the precipitation and runoff, exhibiting extremely high intra-annual and inter-annual 196 variability, mainly occur in the wet season from June to October accounting for almost $65 \%$ of the 197 annual precipitation and over $50 \%$ of the annual runoff, respectively (Zou et al. 2018). Notably, the 198 WRB has been an important agricultural and industrial region in Shaanxi Province, and the runoff has 199 been disturbed by human activities. Thus, the WRB is a suitable candidate to verify the feasibility of 200 proposed model for predicting the non-stationary runoff time series disturbed by climate change and 201 human activities.

Fig.3

The monthly runoff time series employed in this paper were observed from the Zhangjiashan,

204 Zhuangtou and Huaxian stations, which are the main control stations in Jing River, Beiluo River and 205 Wei River, respectively, and data had been collected from 1/1933 to 12/2016, 1/1938 to 12/2016 and $2061 / 1954$ to 12/2016 in the published Hydrological Yearbook, respectively, as shown in Fig.4. It is worth 207 mentioning that the data from Zhangjiashan station is first employed to develop the hybrid model and 208 data from Zhuangtou and Huaxian stations are utilized to further validate the performance of the model.

Tab.1 presents the statistical characteristics of runoff series. It is shown that $\mathrm{Cv}$ values are over

2110.90 in the three stations, and the runoff varies within a relatively large range, and Cs values are greater 212 than zero, indicating that the runoff series have skewness.

Tab.1

\section{$214 \quad 3.2$ Variability analysis of runoff time series}

The monthly runoff hydrographs are plotted in Fig.4, which illustrates drastic intra- and interannual variability and high non-stationary. The cumulative departure curve in Fig.5 was used to 
218 the runoff time series occurred in 1996, 1994 and 1993 at Zhangjiashan, Zhuangtou and Huaxian

219 stations, respectively. Considering that the training period of the model is expected to contain sufficient

220 information on the runoff data after the variability points, the monthly runoff data set is partitioned

221 into the training subset (80\%, before December 2000) and the testing subsets (20\%, from January 2001

222 to December 2016) as shown in Fig.4. In theory, the proposed model could capture variability of the

223 runoff time series during the training period.

\subsection{Decomposition Results with VMD}

The $K$ and $\alpha$, subject to the constrains of the input runoff time series, are two key parameters of

227 VMD algorithm. To achieve satisfactory performance, the center frequency iteration is run to

228 determine the number of the sub-time series $K$, the results show that a convergence of the center

229 frequency of the high frequency sequence is present when $K=8-10$, indicating the decomposition has

230 been completed and $K=8$ is a determined value, which is consistent with earlier study (Huang et al.

231 2016). According to the multiple pre-experiment, $\alpha=1000$ is determined. The default parameters will

232 be adopted for other parameters of the VMD model as shown in Tab.2.

Tab.2

The sub-time series of the original runoff series in Zhangjiashan station are obtained by VMD

235 decomposition, and are shown in Fig.6. 
238 In order to explore the intrinsic characteristics of the runoff variation, each one-dimensional IMF 239 (sub-time series) is reconstructed into a high-dimensional feature space using PSR. Considering the 240 periodic nature of runoff in 12-month, when PSR dimension $d=12$, and delay time $\tau=1$ (Packabd et $241 \mathrm{al} .1980$ ), the $t$-th space vector in the reconstructed phase space is expressed as $X_{t}=\left\{x_{t-1}, x_{t-2}, \mathrm{~L}, x_{t-12}\right\}$ $242 t=13,14, \mathrm{~L}, n$, where, $n$ is the length of the original monthly runoff series, and $x_{t-1}, x_{t-2}, \ldots, x_{t-12}$, 243 respectively, denote 12 different dimensional series, namely 12 candidate input variables series.

244 To avoid the unsatisfactory prediction performance arising from the skewness of runoff data, each

245 candidate input variables series is converted into normal distribution using Box-Cox. The optimal 246 transformation coefficient $\lambda$ is estimated by maximum likelihood method, and the estimated $\lambda$ values 247 of each candidate input variables series for runoff time series are shown as Tab.3.

Tab.3

249 Take the original runoff series as a case to illustrate the normalization of Box-Cox, the probability 250 density of the untransformed and transformed candidate input variables are shown in Fig.7. It can be 251 seen the candidate input variables series transformed by Box-Cox appear the normal distribution.

Fig.7

Fig. 8 is used to analyze the correlation between the output variables and the 12 candidate input

254 variables transformed by Box-Cox. It can be seen that correlation coefficients of the transformed data 255 have been improved, indicating that Box-Cox strengthens the correlation of the data. 
259 which performs well for handling multi-collinearity issue. The LASSO selects significant input 260 variables by adjusting the value of tuning parameter $\rho$ from large to small and controlling the number 261 of non-zero coefficients of candidate input variables (Marami et al., 2016). The importance ranking 262 and the optimal number of selected input variables are shown in the Tab.4. Take the original runoff 263 series as an example, the optimal number of selected input variables is 4 using trial and error method, 264 thus the input variables are $x_{t-1}, x_{t-12}, x_{t-11}, x_{t-2}$.

Tab.4

\section{6 Development and training of the forecasting models}

In order to verify the performance of the VMD-BC-Elman model, single models and other

combinations of models are used for comparison in Fig.9. The training of these models will be described in this section.

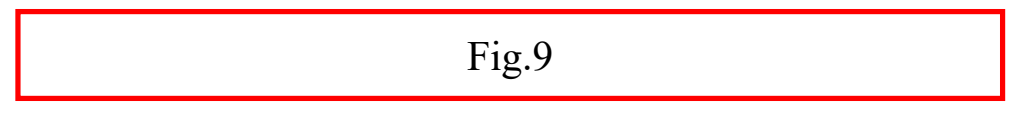

\section{Group 1: Single models}

The standard Elman and SVM are individually used as basic models for developing the hybrid

273 models. The activation functions and parameters of Elman are set utilizing the training data sets with

274 the maximum number of the training as 500, the target error 0.001 , and the learning coefficient 0.01 .

275 The hidden layer activation function employs Tansig, the output layer activation function uses Purelin,

276 and the training algorithm adopts L-M algorithm. The number of each layer is determined using the 
277 trial-and-error method, and the trained Elman architecture is presented in Tab.5. In SVM model, the 278 radial basis kernel function is selected with insensitivity loss parameter at 0.01 , penalty factor at 1 and 279 kernel function parameter at 2.

$280 \quad$ Group 2: Decomposition-based hybrid models

281 Decomposition-based hybrid models are developed by coupling EMD, EEMD and VMD with 282 Elman or SVM, respectively. The original runoff series are decomposed into 6, 11 and 8 sub-time 283 series by EMD, EEMD and VMD, respectively. Each sub-time series are modelled using the Elman or 284 SVM. In Elman training, the target error is set at 0.00001, and the remaining parameters and the 285 activation functions are constants as the single model have in Group 1. The three-layer architecture of 286 each sub-time series is determined by trial and error method, and the optimal number of the input, with 287 hidden and output neurons listed in Tab.5. For SVM training, the number of the input variables of each 288 sub-time series is selected in Tab.4 and the other parameters are the same with those of the standard 289 SVM.

\section{Tab.5}

293 developed by introducing Box-Cox into Elman or SVM, respectively. The input variables series are 294 transformed into normal distribution using Box-Cox with the parameters $\lambda$ in Tab.3. The model 295 architecture and parameters are the same as the single models.

\section{Group 3: Box-Cox-based hybrid models}

To reduce the negative effect of the skewed runoff data on the modeling, BC-based models are 
299 proposed by introducing Box-Cox and VMD into the Elman or SVM. In this section, the VMD-BC-

300 Elman architecture of each sub-time series is the same as those of VMD-Elman, and the VMD-BC-

301 SVM modeling parameters are the same as those of SVM.

\subsection{Results and discussion for Zhangjiashan station}

Based on the training models above, runoff time series are modelled using the different models,

respectively. And the performance statistical metrics are listed in the Tab.6. The hybrid models are

305 shown to be more accurate than their standalone counterparts; and the performance of the VMD-BC-

306 Elman is best in all models.

In the standalone models, Elman and SVM have unsatisfactory predicting results with NSE $<0.6$,

308 lower R and higher NMSE and PBIAS as shown in the Tab.6. Compared to SVM, the Elman provides

309 better general results in training period because its recurrent feedback networks with short-term

310 memory increase the ability to process dynamic information. However, the performance of the Elman

311 is unstable, which may lead to over-fitting. And the standalone SVM, which is based on the risk

312 minimization principle, has more robustness and generalizability for estimating non-stationary and

313 skewed time series than the Elman does. The Elman model provides better predicting results in training

314 period and SVM provides better predicting results in testing period, which is consistent with other

315 studies (Song et al. 2020; Feng et al. 2020).

Compared with the standalone models, the metric values of decomposition-based hybrid models

are satisfactory, as shown in the Tab.6. The six models yield $\mathrm{R}$ values in the range of 0.7878 to 0.9843 , 
319 from $1.26 \%$ to $19.26 \%$ in the testing period, thus the decomposition-based hybrid models are superior 320 to the counterpart single models (Napolitano et al. 2011; Mohammad et al. 2019). The metric values 321 of VMD-Elman are best in six models which indicates the VMD-Elman outperforms the other five 322 hybrid models and the VMD has a better ability to denoise the runoff data than EMD and EEMD do, 323 which agrees with the results of other researchers who have modelled runoff time series based on EMD 324 EEMD and VMD decomposition (Xie et al. 2019; Zuo et al. 2020).

In order to eliminate skewness of runoff time series, BC-based models are introduced. The Tab.6

326 shows that the statistical metrics of the BC-SVM and BC-Elman models are much better than those of

327 the single models. Taking NMSE in the testing period as an example, the NMSE for the BC-Elman, 328 BC-SVM, Elman and SVM models are 18.3\%, 24.84\%, 92.91\% and 66.93\%, respectively. This

329 illustrates that the performance of the BC-based hybrid models is superior to the single models, and 330 BC-Elman is better than BC-SVM. The excellent performance of BC-based models in this paper is 331 similar to the study on hydrology frequency analysis, which shows that Box-Cox is capable of 332 providing robustness in fitting models (Seong 2014).

The statistical metrics of the VMD-BC-Elman and VMD-BC-SVM are shown in the Tab.6. In the

334 testing period, the R of the VMD-BC-Elman are $0.04 \%, 6.05 \%$, and $0.14 \%$ greater than that of VMD-

335 Elman, BC-Elman and VMD-BC-SVM models; its NSE is $0.08 \%, 17.65 \%$, and $0.39 \%$ greater 336 respectively; its NMSE is $1.97 \%, 78.25 \%$, and $8.50 \%$ smaller, and its PBIA is $68.29 \%, 96.54 \%$, and $33746.58 \%$ smaller, respectively. The statistical metrics values demonstrate the fact that, although VMD 338 or Box-Cox alone can improve the ability of a single Elman, integration of VMD and Box-Cox into 339 Elman can promote their predicting to a new level with the VMD-BC-Elman outperforming the VMD- 
340 BC-SVM. Considering these results, it is evident that the proposed VMD-BC-Elman has more stable

341 and consistent performance. It is affirmed that the AI models, combined the VMD and Box-Cox,

342 represent a significant improvement in runoff prediction.

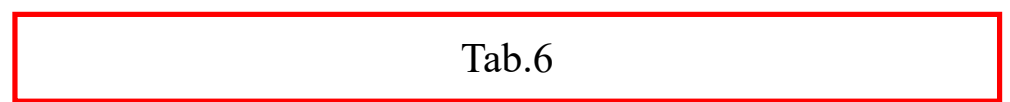

344 While the statistical metrics can evaluate the overall performance of models, hydrographs and

345 scatter plots can further identify the temporal correspondence. Fig.10-11 display the hydrographs and

346 scatter plots of the observed and predicted runoff from the different models in the testing periods. In

347 Fig.10 (a) and Fig. 11(a)-(b), the single models are not able to accurately capture the observed runoff,

348 especially for the peak flow, and the scatter plots of the observed and estimated runoff are more

349 dispersed than the hybrid models. Notably, in Fig.10(b)-(c) and Fig.11(c)-(j), the estimated values

350 generated by the decomposition-based and BC-based hybrid models are closer to the corresponding

351 observed values and the scatter plots are clustered closer to the ideal fit. In Fig.10(d) and Fig.11(k)-(l),

352 it is also obvious that VMD-BC-Elman and VMD-BC-SVM have a greater accuracy as the scatter plots

353 are concentrated closer to the ideal fitting line, illustrating the VMD-BC-based hybrid models are more

354 effective in estimating the peak values of data than other models. Particularly, the VMD-BC-Elman

355 has the best capability to capture the information on the overall features of runoff time series. Therefore,

356 it is also reaffirmed that the VMD and Box-Cox can effectively improve the stability and consistency

357 of the models, and the VMD-BC-Elman exhibits the strongest performance.

Fig. 10

Fig.11

In Fig.12, the boxplot diagram is designed to depict the errors distribution between estimated and 
361 observed values in the entire tested dataset in order to further verify the prediction performance of all

362 the models. It can be seen from Fig.12 that the predicted errors including the first, median and third

363 quartiles, minimum and maximum non-outlier are smallest when the VMD-BC-Elman is employed

364 compared with the compared models. It indicates that the VMD and Box-Cox enhance the robustness

365 of the models, and the VMD-BC-Elman is superior to the other models, which is consistent with the

366 above findings.

Fig.12

The Taylor diagram as shown in Fig.13, integrating multiple characteristics of models into a

compact plot (Taylor 2001), can present the performance of the models by a comprehensive way. It

shows how close or faraway the simulated values of each model to or from the observed runoff data

in the testing period, quantified via the correlation coefficient, standard deviation and root mean square

error in Taylor diagram. It is evident that VMD-BC-Elman is the closest to the observed reference

point with the lowest RMSD, the highest correlation coefficient and the lower SD, following by the

374 VMD-BC-based and the VMD-based models while the single models are positioned the furthest from

375 the reference point. Obviously, the VMD-BC-Elman performs best for monthly runoff prediction,

376 which can be explained that the provision of the VMD and Box-Cox contributes to the improved

377 stability and consistency of the models, and avoid over-fitting.

Fig.13

\subsection{Results and discussion for Zhuangtou and Huaxian station}

In order to further verify the feasibility of the VMD-BC-Elman, the runoff at Zhuangtou and

381 Huaxian stations are modelled. In Tab.7 and Tab.8, the hybrid models are better than the single models 
382 in the testing period. The VMD-based models are generally more accurate and stable than the EMD-

383 based and the EEMD-based models. Meanwhile, the BC-based models outperform the single models.

384 Obviously, the VMD-BC-Elman and VMD-BC-SVM outperform the VMD-based and the BC-based 385 models, and the VMD-BC-Elman is superior to the VMD-BC-SVM which is similar to the results of 386 Zhangjiashan station, so it is safe to conclude that the VMD-BC-Elman has the best forecast 387 performance in this paper.

Tab.7

In Fig.14, the proposed hybrid VMD-BC-Elman exhibits the best fit for the observed runoff 391 compared with other models, and estimates by the VMD-BC-Elman are closest to the corresponding 392 observed values and follow the same trend in all plots. Their scatter plots are closest to the ideal line, 393 especially around the peak flow, which also indicates that the VMD-BC-Elman has best predictive 394 skills. In conclusion, the VMD-BC-Elman exhibits the best performance in terms of the accuracy, 395 stability and consistency, and the combination of VMD and Box-Cox has great potential in monthly 396 runoff prediction areas.

To estimate non-stationary and skewed monthly runoff series, a novel VMD-BC-Elman model 400 was developed in this paper and runoff data in Zhangjiashan, Zhuangtou and Huaxian stations in the 401 Wei River Basin were used. The steps of this model are as follows: (1) The original non-stationary 402 monthly runoff time series were decomposed into a set of relatively stationary sub-time series IMFs 
403 by VMD. (2) Each IMF was reconstructed into a 12-dimensional phase space by PSR, and each 404 candidate input variables series was normalized using Box-Cox. (3) LASSO was employed to 405 determine the optimal number of the input variables. (4) Each sub-time series was simulated by the 406 Elman. The final results are generated by summing up the estimates of all the sub-time series.

407 The proposed VMD-BC-Elman exhibited the best precision, stability and consistency for 408 predicting the non-stationary and skewed monthly runoff series. The remarkable advantages were 409 demonstrated in the following aspects: (1) The proposed model with a perfect denoising ability, can 410 effectively distinguish the modalities of the complex non-stationary series, and adaptably decompose 411 the original series into the relative stationary sub-time series using the VMD. (2) It can remove the 412 skewness of the original runoff series using Box-Cox, and convert the skewed candidate input variables 413 into the normal distribution, which can enhance the correlation between the input and output variables, 414 and improve the mapping ability and self-learning ability of the Elman. (3) Integrating decomposition415 normalization- simulation- reconstruction techniques together, VMD-BC-Elman can effectively 416 identify and separate the different modal features, distinguish and construct the different distribution 417 features of the runoff time series, and accurately sum up the optimal estimates of sub-time series to 418 achieve the better prediction for the monthly runoff. Overall, the VMD-BC-Elman represents an 419 effective tool for forecasting non-stationary and skewed monthly runoff series.

In spite of the remarkable performance of the VMD-BC-Elman over comparative models, the 421 study has limitations that create opportunities for further research. Firstly, the prediction results of high 422 IMF are unsatisfactory possibly due to too much noise. Secondly, the proposed model only considers 423 one-step prediction, and multi-step prediction has not been discussed yet. Finally, the proposed model 424 does not take in account the physical mechanism of runoff formation. The following aspects are worthy 425 of further research. Firstly, the high- and low-IMFs are expected to develop different models to more 
426 effectively predict the different frequency characteristics. Secondly, it would be useful to explore a 427 model utilizing multi-step prediction approach. Thirdly, physically-based hydrology model can be 428 introduced into the current VMD-BC-Elman, so this data-driven model can combine the strength of 429 self-learning ability with enhanced physical mechanism.

\section{Declarations}

431 Acknowledgements This research was funded by Science-Technology Plan Program of Water 432 Conservancy Fund of Shaanxi Province, grant number 2019slkj-14, and National Natural Science 433 Foundation of China under grant 5149222, 52079110. Sincere gratitude is extended to the editor and 434 anonymous reviewers for their professional comments and corrections.

435 Conflict of Interest The authors declared that they have no conflicts of interest to this work.

436 Availability of data and material The data used to support the findings of this study are available 437 from the corresponding author upon reasonable request.

438 Code availability The code used to support the findings of this study are available from the 439 corresponding author upon reasonable request.

440 Authors' Contributions Fangqin Zhang: Investigation, Modeling, Calculation, Writing-Original 441 Draft. Yan Kang: Conceptualization, Methodology, Writing-Review \& Editing, Supervision. Xiao 442 Cheng: Investigation, Data curation. Peiru Chen: Investigation. Songbai Song: Writing-Review \& 443 Editing.

444 Ethics Approval Not applicable.

445 Consent to Participate Not applicable.

446 Consent to Publication Not applicable. 


\section{References}

448 Aggarwal SK, Goel A, Singh VP (2012). Stage and discharge forecasting by SVM and ANN techniques. $449 \quad$ Water Resource Management 26 (13): 3705-3724.

450 Barge JT, Sharif HO (2016). An ensemble empirical mode decomposition, self-organizing map, and 451 linear genetic programming approach for forecasting river streamflow. Water 8(2):247.

452 Bojang PO, Yang TC, Pham QB (2020). Linking Singular Spectrum Analysis and Machine Learning 453 for Monthly Rainfall Forecasting. Applied Sciences-Basel 10(09).

454 Box GEP, Cox, DR (1964). An analysis of transformations. Journal of the Royal Statistical Society 455 B26: 211-252.

456 Chandra R (2015). Competition and Collaboration in Cooperative Coevolution of Elman Recurrent Neural Networks for Time-Series Prediction. IEEE Transactions on Neural Networks and

Deb S, Dandapat S, Krajewski (2020). Analysis and Classification of Cold Speech Using Variational Mode Decomposition. IEEE Transactions on Affective Computing 11(02):296-307.

464 Demirel MC, Venancio A, Kahya E, 2009. Flow forecast by SWAT model and ANN in Pracana basin, Portugal. Advances in Engineering Software 40(07):467-473.

466 Dragomiretskiy K, Zosso D (2014). Variational mode decomposition. IEEE Trans Signal Process 467 $62(3): 531-544$. 
468 Feng ZK, Niu WJ, Tang ZY, Jiang ZQ, Xu Y, Liu Y, Zhang HR (2020). Monthly runoff time series 469 prediction by variational mode decomposition and support vector machine based on quantum$470 \quad$ behaved particle swarm optimization. Journal of Hydrology 583.

471 Hamasaki T, Kim SY (2017). Box and Cox power-transformation to confined and censored non-normal 472 responses in regression. Computational Statistics \&Data Analysis 51(8):3788-3799.

473 Himanshu SK, Pandey A, Yadav B (2017). Ensemble Wavelet-Support Vector Machine Approach for 474 Prediction of Suspended Sediment Load Using Hydrometeorological Data. Journal of Hydrologic

476 Huang N, Chen H, Cai G, Fang L, Wang Y (2016). Mechanical fault diagnosis of high voltage circuit 477 478 Huang NE, Wu MLC, Long SR, Shen SSP, Qu WD, Gloersen P, Fan KL (2003). A confidence limit

Jiang RG, Wang YP, Xie JC, Zhao Y, Li FW, Wang XJ (2019). Assessment of extreme precipitation

Karamouz M, Ahmadi A, Moridi A (2009). Probabilistic reservoir operation using Bayesian stochastic model and support vector machine. Advances in Water Resources 32(11):1588-1600. events and their teleconnections to El Nino southern oscillation, a case study in the Wei River basin of China. Atmospheric Research 218:372-384.

model and support vector machine. Advances in Water Resources 32(11):1588-1600.

Krishnan S, Lokesh S, Devi MR (2019). An efficient Elman neural network classifier with cloud supported internet of things structure for health monitoring system. Computer Networks 14(5):201-210. 
489 Kuremoto T, Kimura S, Kobayashi K, Obayashi M (2014). Time series forecasting using a deep belief 490 network with restricted Boltzmann machines. Neurocomputing 137: 47-56.

491 Li H, Fan BJ, Jia R, Zhai F, Bai L, Luo XQ (2020). Research on multi-domain fault diagnosis of 492 gearbox of wind turbine based on adaptive variational mode decomposition and extreme learning 493 machine algorithms. Energies 13(6):1375.

494 Li XY, Zhang L, Wang ZP, Dong P (2019). Remaining useful life prediction for lithium-ion batteries 495 based on a hybrid model combining the long short-term memory and Elman neural networks. $496 \quad$ Journal of Energy Storage 21:510-518.

497 Mehrgini B, Izadi H, Memarian H (2019). Shear wave velocity prediction using Elman artificial neural 498

499 Moeeni H, Bonakdari H, Fatemi SE (2017). Stochastic model stationarization by eliminating the 500 periodic term and its effect on time series prediction. Journal of Hydrology 547:348-364.

501 Mohamad JA, Mohamad RK, Ozgur K, Vahid N (2017). A new approach for simulating and forecasting 502 the rainfall-runoff process within the next two months. Journal of Hydrology 548(4):588-597.

Mohanty S, Gupta KK, Raju KS (2018). Hurst based vibro-acoustic feature extraction of bearing using EMD and VMD. Measurement 117:200-220.

Myronidis D, Ioannou K, Fotakis D, Dorflinger G (2018). Streamflow and hydrological drought trend analysis and forecasting in Cyprus. Water Resources Management 32(5):1759-1776.

507 Napolitano G, Serinaldi F, See L (2011). Impact of EMD decomposition and random initialisation of 508 weights in ANN hindcasting of daily stream flow series: An empirical examination. Journal of 509 Hydrology 406 (3):199-214. 
510 Nash J, Sutcliffe J (1970). River flow forecasting through conceptual models part I—a discussion of 511 principles. Journal of Hydrology 10(3): 282-290.

512 Packard NH, Crutchfieid JP, Farmer JD, et al (1980). Geometry from a time series. Physical Review $513 \quad$ Letters 45:712.

514 Partington D, Brunner P, Simmons CT, Werner AD, Therien R, Maier HP, Dandy GC (2012). 515 Evaluation of outputs from automated baseflow separation methods against simulated baseflow 516 from a physically based, surface water-groundwater flow model. Journal of Hydrology 458: 28 39.

518 Peng Y, Wang GL, Tang GL, Zhou HC, Wang YJ, Jian DP (2011). Study on reservoir operation optimization of Ertan hydropower Station considering GFS forecasted precipitation. Science

521 Sankaran A, Reddy MJ (2016). Analyzing the hydroclimatic teleconnections of summer monsoon rainfall in Kerala, India, using multivariate empirical mode decomposition and time-dependent 524 Sarlak N (2008). Annual streamflow modelling with asymmetric distribution function. Hydrological Processes 22(17):3403-3409.

526 Sedki A, Quazar D, EI Mazoudi E (2008). Evolving neural network using real coded genetic algorithm 527 for daily rainfall-runoff forecasting. Expert Systems with Applications 36:4523-4527.

528 Seong KW (2014). Deriving a practical form of IDF formula using transformed rainfall intensities. Hydrological Processes 28(06):2881-2896.

530 Song PB, Liu WF, Sun JH, Wang C, Kong LZ, Nong ZX, Lei XH, Wang H (2020). Annual Runoff 

Ganjiang River Basin. Water 12(08).

Taylor KE (2001). Summarizing multiple aspects of model performance in a single diagram. Journal of Geophysical Research-Atmospheres 106 (D7):7183-7192.

535 Tiwari MK, Adamowaki J (2013). Urban water demand forecasting and uncertainty assessment using ensemble wavelet-bootstrap-neural network models. Water resources research 49(10):6486-6507.

537 Vasiliades L, Loukas A (2009). Hydrological response to meteorological drought using the Palmer drought indices in Thessaly, Greece. Desalination 237(1-3):3-21.

539 Wang QY, Liu Y, Yue QM, Zheng YX, Yao XL, Yu JS (2020). Impact of Input Filtering and 540 Architecture Selection Strategies on GRU Runoff Forecasting: A Case Study in the Wei River Basin, Shaanxi, China. Water 12(12).

Wang WC, Xu DM, Chau KW, Chen S (2013). Improved annual rainfall-runoff forecasting using PSOSVM model based on EEMD. Journal of Hydroinform, 15(4):1377-1390.

Wen XH, Feng Q, Deo RC, et al (2019). Two-phase extreme learning machines integrated with the complete ensemble empirical mode decomposition with adaptive noise algorithm for multi-scale runoff prediction problems. Journal of Hydrology 570:167-184.

Wu Z, Huang NE (2009). Ensemble empirical mode decomposition: A noise-assisted data analysis

Xie T, Zhang G, Hou JW, Xie JC, Lv M, Liu FC (2019). Hybrid forecasting model for non-stationary daily runoff series: A case study in the Han River Basin, China. Journal of Hydrology 577(10). 

an Improved Ensemble Empirical Mode Decomposition (EEMD) Model. Water 10(04).

553 Zhang H, Singh VP, Wang B, Yu Y (2016). CEREF: a hybrid data-driven model for forecasting annual 554 streamflow from a socio-hydrological system. Journal of Hydrology 540:246-256.

555 Zhang X, Peng Y, Zhang C, Wang B (2015). Are hybrid models integrated with data preprocessing 556 techniques suitable for monthly streamflow forecasting? Some experiment evidences. Journal of $557 \quad$ Hydrology 530: 137-152.

558 Zou L, Xia J, She DX (2018). Analysis of impacts of climate change and human activities on 559 hydrological drought: a case study in the Wei River basin, China. Water Resources Management $560 \quad 32(4): 1421-1438$.

561 Zuo GG, Luo JG, Wang N, et al (2020). Decomposition ensemble model based on variational mode 562 decomposition and long short-term memory for streamflow forecasting. Journal of Hydrology $563 \quad 585(06)$ 


\section{Table Caption}

566 Tab.1 Profiles of hydrological stations and statistical characteristics of the runoff series at Zhangjiashan,

567 Zhuangtou and Huaxian stations.

568 Tab.2 Parameters of VMD determined in the iteration experiments.

569 Tab.3 The $\lambda$ values of each candidate input variables series for the original runoff series and each

570 IMF at Zhangjiashan station.

571 Tab.4 Importance ranking of the selected input variables for original series and sub-time series

572 decomposed at Zhangjiashan station.

573 Tab.5 Elman predicted architecture of the original and each sub-time series based on different

574 decomposition methods at Zhangjiashan Station.

575 Tab.6 Statistical metrics for models' performance evaluation in the training and testing periods at 576 zhangjiashan station.

577 Tab.7 As in Tab.6, but for Zhuangtou station.

578 Tab.8 As in Tab.6, but for Huaxian station. 
579 Tab.1 Profiles of hydrological stations and statistical characteristics of the runoff series at Zhangjiashan,

580 Zhuangtou and Huaxian stations.

\begin{tabular}{|c|c|c|c|c|c|c|c|c|c|c|}
\hline Station & Longitude & Latitude & $\begin{array}{c}\text { Control } \\
\text { Area }\left(\mathrm{km}^{2}\right)\end{array}$ & Data Sets & Data Length & $\begin{array}{c}\operatorname{Max} \\
\left(\mathrm{m}^{3} / \mathrm{s}\right)\end{array}$ & $\underset{\left(\mathrm{m}^{3} / \mathrm{s}\right)}{\operatorname{Min}}$ & $\begin{array}{l}\text { Mean } \\
\left(\mathrm{m}^{3} / \mathrm{s}\right)\end{array}$ & $\mathrm{Cv}$ & Cs \\
\hline \multirow{3}{*}{ Zhangjiashan } & \multirow{3}{*}{$108^{\circ} 36^{\prime}$} & \multirow{3}{*}{$34^{\circ} 38^{\prime}$} & \multirow{3}{*}{43,216} & All & $1933-2016$ & 676.00 & 1.00 & 50.12 & 1.17 & 3.64 \\
\hline & & & & Training & $1933-2000$ & 676.00 & 1.00 & 54.07 & 1.16 & 3.44 \\
\hline & & & & Testing & 2001-2016 & 213.00 & 7.00 & 33.34 & 0.97 & 3.42 \\
\hline \multirow{3}{*}{ Zhuangtou } & \multirow{3}{*}{$109^{\circ} 50^{\prime}$} & \multirow{3}{*}{$35^{\circ} 02^{\prime}$} & \multirow{3}{*}{25,154} & All & $1938-2016$ & 199.00 & 2.00 & 24.86 & 0.90 & 3.19 \\
\hline & & & & Training & $1938-2000$ & 199.00 & 2.00 & 26.41 & 0.88 & 3.07 \\
\hline & & & & Testing & $2001-2016$ & 138.00 & 3.00 & 18.75 & 0.90 & 3.94 \\
\hline \multirow{3}{*}{ Huaxian } & \multirow{3}{*}{$109^{\circ} 46^{\prime}$} & \multirow{3}{*}{$34^{\circ} 35^{\prime}$} & \multirow{3}{*}{106,498} & All & $1954-2016$ & 1690.00 & 2.70 & 206.65 & 1.15 & 2.69 \\
\hline & & & & Training & $1954-2000$ & 1690.00 & 2.70 & 224.02 & 1.13 & 2.50 \\
\hline & & & & Testing & $2001-2016$ & 1250.00 & 8.59 & 156.71 & 1.14 & 3.71 \\
\hline
\end{tabular}

581 Max, Min, Mean, $\mathrm{Cv}$ and Cs represent maximum, minimum, mean value, coefficient of variation and coefficient of skewness of data

582 sets, respectively.

583 
584 Tab.2 Parameters of VMD determined in the iteration experiments.

585

\begin{tabular}{ccccccc}
\hline Parameters & $\alpha$ & $\tau$ & $\mathrm{K}$ & $\mathrm{DC}$ & Init & tol \\
\hline Values & 1000 & 0 & 8 & 0 & 1 & $10^{-7}$ \\
\hline
\end{tabular}


586 Tab.3 The $\lambda$ values of each candidate input variables series for the original runoff series and each 587 IMF at Zhangjiashan station.

\begin{tabular}{c|cccccccccccc}
\hline & $x_{t-1}$ & $x_{t-2}$ & $x_{t-3}$ & $x_{t-4}$ & $x_{t-5}$ & $x_{t-6}$ & $x_{t-7}$ & $x_{t-8}$ & $x_{t-9}$ & $x_{t-10}$ & $x_{t-11}$ & $x_{t-12}$ \\
\hline Original & 0.2104 & 0.2112 & 0.2112 & 0.2111 & 0.2109 & 0.2107 & 0.2108 & 0.2108 & 0.2114 & 0.2126 & 0.2126 & 0.2129 \\
IMF1 & 0.2723 & 0.2796 & 0.2873 & 0.2953 & 0.3035 & 0.3118 & 0.3199 & 0.3276 & 0.3351 & 0.3420 & 0.3483 & 0.3536 \\
IMF2 & 0.8304 & 0.8323 & 0.8345 & 0.8361 & 0.8359 & 0.8347 & 0.8343 & 0.8351 & 0.8352 & 0.8339 & 0.8333 & 0.8353 \\
IMF3 & 1.0887 & 1.0871 & 1.0867 & 1.0869 & 1.0873 & 1.0881 & 1.0890 & 1.0891 & 1.0892 & 1.0899 & 1.0893 & 1.0909 \\
IMF4 & 0.9419 & 0.9395 & 0.9384 & 0.9401 & 0.9434 & 0.9465 & 0.9473 & 0.9454 & 0.9458 & 0.9434 & 0.9460 & 0.9363 \\
IMF5 & 1.1262 & 1.1273 & 1.1256 & 1.1247 & 1.1249 & 1.1259 & 1.1270 & 1.1276 & 1.1264 & 1.1267 & 1.1257 & 1.1268 \\
IMF6 & 0.7677 & 0.7674 & 0.7678 & 0.7686 & 0.7678 & 0.7680 & 0.7690 & 0.7685 & 0.7679 & 0.7661 & 0.7653 & 0.7658 \\
IMF7 & 1.1633 & 1.1634 & 1.1633 & 1.1631 & 1.1638 & 1.1630 & 1.1640 & 1.1635 & 1.1630 & 1.1636 & 1.1666 & 1.1659 \\
IMF8 & 1.1784 & 1.1779 & 1.1789 & 1.1778 & 1.1792 & 1.1792 & 1.1788 & 1.1813 & 1.1801 & 1.1809 & 1.1822 & 1.1812 \\
\hline
\end{tabular}


589 Tab.4 Importance ranking of the selected input variables for original series and sub-time series

590 decomposed at Zhangjiashan station.

\begin{tabular}{cllc}
\hline No. & Series & \multicolumn{1}{c}{ Raking the selected input variables } & Number of input variables \\
\hline 1 & Original & $x_{t-1}, x_{t-12}, x_{t-11}, x_{t-2}, x_{t-10}, x_{t-5}, x_{t-4}, x_{t-3}, x_{t-9}, x_{t-6}, x_{t-8}, x_{t-7}$ & 4 \\
2 & IMF1 & $x_{t-12}, x_{t-11}, x_{t-10}, x_{t-9}, x_{t-8}, x_{t-7}, x_{t-6}, x_{t-5}, x_{t-4}, x_{t-3}, x_{t-2}, x_{t-1}$ & 3 (EEMD:6) \\
3 & IMF2 & $x_{t-9}, x_{t-3}, x_{t-10}, x_{t-8}, x_{t-4}, x_{t-2}, x_{t-11}, x_{t-12}, x_{t-7}, x_{t-5}, x_{t-6}, x_{t-1}$ & 7 \\
4 & IMF3 & $x_{t-2}, x_{t-6}, x_{t-10}, x_{t-11}, x_{t-12}, x_{t-7}, x_{t-9}, x_{t-5}, x_{t-8}, x_{t-3}, x_{t-1}, x_{t-4}$ & 8 \\
5 & IMF4 & $x_{t-11}, x_{t-10}, x_{t-8}, x_{t-7}, x_{t-4}, x_{t-5}, x_{t-1}, x_{t-2}, x_{t-12}, x_{t-9}, x_{t-6}, x_{t-3}$ & 8 \\
6 & IMF5 & $x_{t-11}, x_{t-1}, x_{t-9}, x_{t-12}, x_{t-3}, x_{t-7}, x_{t-5}, x_{t-10}, x_{t-8}, x_{t-6}, x_{t-4}, x_{t-2}$ & 8 \\
7 & IMF6 & $x_{t-12}, x_{t-10}, x_{t-11}, x_{t-7}, x_{t-9}, x_{t-4}, x_{t-8}, x_{t-1}, x_{t-5}, x_{t-6}, x_{t-2}, x_{t-3}$ & 8 (EMD:10) \\
8 & IMF7 & $x_{t-12}, x_{t-11}, x_{t-10}, x_{t-8}, x_{t-9}, x_{t-7}, x_{t-3}, x_{t-2}, x_{t-6}, x_{t-4}, x_{t-5}, x_{t-1}$ & 8 \\
9 & IMF8 & $x_{t-7}, x_{t-8}, x_{t-6}, x_{t-9}, x_{t-12}, x_{t-10}, x_{t-11}, x_{t-5}, x_{t-4}, x_{t-3}, x_{t-2}, x_{t-1}$ & 8 \\
\hline
\end{tabular}


592 Tab.5 Elman predicted architecture of the original and each sub-time series based on different

593 decomposition methods at Zhangjiashan Station.

\begin{tabular}{ccccc}
\hline \multirow{2}{*}{ Series } & Single model & \multicolumn{3}{c}{ Hybrid models with different decomposition methods } \\
\cline { 2 - 5 } & Elman & EMD-Elman & EEMD-Elman & VMD-Elman \\
\hline Original & $4-12-1$ & - & - & - \\
IMF1 & - & $3-15-1$ & $6-15-1$ & $3-10-1$ \\
IMF2 & - & $7-13-1$ & $7-13-1$ & $7-13-1$ \\
IMF3 & - & $8-13-1$ & $8-13-1$ & $8-13-1$ \\
IMF4 & - & $8-13-1$ & $8-13-1$ & $8-13-1$ \\
IMF5 & - & $8-13-1$ & $8-13-1$ & $8-13-1$ \\
IMF6 & - & $10-13-1$ & $8-13-1$ & $8-13-1$ \\
IMF7 & - & - & $8-13-1$ & $8-13-1$ \\
IMF8 & - & - & $8-13-1$ & $8-13-1$ \\
IMF9 & - & - & $8-13-1$ & - \\
IMF10 & - & - & $8-13-1$ & - \\
IMF1 & - & - & $8-13-1$ & - \\
\hline
\end{tabular}


595 Tab.6 Statistical metrics for models' performance evaluation in the training and testing periods at 596 zhangjiashan station.

\begin{tabular}{|c|c|c|c|c|c|c|c|c|c|}
\hline \multirow{2}{*}{ Types } & \multirow{2}{*}{ Models } & \multicolumn{4}{|c|}{ Training Dataset } & \multicolumn{4}{|c|}{ Testing Dataset } \\
\hline & & $R$ & NSE & $N M S E \%$ & PBIAS\% & $R$ & NSE & $N M S E \%$ & PBIAS\% \\
\hline \multirow{2}{*}{ Original } & SVM & 0.6001 & 0.3229 & 67.63 & 18.39 & 0.5756 & 0.3272 & 66.93 & 5.83 \\
\hline & Elman & 0.7395 & 0.5468 & 45.26 & -0.53 & 0.5954 & 0.0660 & 92.91 & -21.18 \\
\hline \multirow{6}{*}{ Decomposition } & EMD-SVM & 0.7735 & 0.5758 & 42.37 & 9.62 & 0.7878 & 0.5754 & 42.24 & 19.26 \\
\hline & EEMD-SVM & 0.8772 & 0.7646 & 23.51 & -3.25 & 0.9063 & 0.8028 & 19.62 & -12.97 \\
\hline & VMD-SVM & 0.9819 & 0.9524 & 4.75 & 0.43 & 0.9830 & 0.9552 & 4.45 & 1.81 \\
\hline & EMD-Elman & 0.8983 & 0.8059 & 19.38 & -0.94 & 0.8239 & 0.6371 & 36.10 & 2.65 \\
\hline & EEMD-Elman & 0.9644 & 0.9301 & 6.98 & 0.04 & 0.9162 & 0.8200 & 17.91 & -11.43 \\
\hline & VMD-Elman & 0.9836 & 0.9580 & 4.19 & 0.13 & 0.9843 & 0.9592 & 4.06 & 1.23 \\
\hline \multirow{2}{*}{$\begin{array}{l}\text { Box-Cox } \\
\text { transformation }\end{array}$} & BC-SVM & 0.8674 & 0.7328 & 26.69 & 9.47 & 0.9059 & 0.7503 & 24.84 & 7.42 \\
\hline & BC-Elman & 0.9409 & 0.8847 & 11.52 & -2.81 & 0.9285 & 0.8160 & 18.30 & 11.28 \\
\hline \multirow{2}{*}{$\begin{array}{l}\text { Decomposition } \\
\text { and Box-Cox }\end{array}$} & VMD-BC-SVM & 0.9826 & 0.9537 & 4.63 & 0.19 & 0.9833 & 0.9563 & 4.35 & 0.73 \\
\hline & VMD-BC-Elman & 0.9844 & 0.9596 & 4.03 & 0.10 & 0.9847 & 0.9600 & 3.98 & 0.39 \\
\hline
\end{tabular}


Tab.7 As in Tab.6, but for Zhuangtou station.

\begin{tabular}{|c|c|c|c|c|c|c|c|c|c|}
\hline \multirow[b]{2}{*}{ Types } & \multirow[b]{2}{*}{ Model } & \multicolumn{4}{|c|}{ Training dataset } & \multicolumn{4}{|c|}{ Testing Dataset } \\
\hline & & $R$ & $N S E$ & $\begin{array}{c}\text { NMSE } \\
\%\end{array}$ & $P B I A S \%$ & $R$ & $N S E$ & $N M S E \%$ & PBIAS\% \\
\hline \multirow[b]{2}{*}{ Original } & SVM & 0.5693 & 0.2888 & 71.02 & 15.33 & 0.4928 & 0.2300 & 76.6 & 8.05 \\
\hline & Elman & 0.7029 & 0.4940 & 50.53 & 0.16 & 0.3656 & 0.0588 & 93.63 & -3.86 \\
\hline \multirow{6}{*}{ Decomposition } & EMD-SVM & 0.7756 & 0.5862 & 41.32 & 7.70 & 0.7665 & 0.5545 & 44.31 & -15.74 \\
\hline & EEMD-SVM & 0.8959 & 0.8007 & 19.90 & 2.60 & 0.9224 & 0.8424 & 15.68 & -6.98 \\
\hline & VMD-SVM & 0.9807 & 0.9495 & 5.04 & 0.03 & 0.9803 & 0.9445 & 5.52 & -1.78 \\
\hline & EMD-Elman & 0.9150 & 0.8372 & 16.26 & -0.76 & 0.7889 & 0.4510 & 54.62 & 34.79 \\
\hline & EEMD-Elman & 0.9580 & 0.9170 & 8.29 & 1.17 & 0.9202 & 0.8353 & 16.39 & -3.12 \\
\hline & VMD-Elman & 0.9829 & 0.9560 & 4.40 & -0.09 & 0.9800 & 0.9483 & 5.15 & 0.18 \\
\hline \multirow{2}{*}{$\begin{array}{c}\text { Box-Cox } \\
\text { transformation }\end{array}$} & BC-SVM & 0.7999 & 0.6202 & 37.93 & 0.51 & 0.7840 & 0.5757 & 42.21 & 7.45 \\
\hline & BC-Elman & 0.9304 & 0.8656 & 13.43 & 0.01 & 0.8029 & 0.6285 & 36.96 & 9.25 \\
\hline \multirow{2}{*}{$\begin{array}{l}\text { Decomposition } \\
\text { and Box-Cox }\end{array}$} & VMD-BC-SVM & 0.9815 & 0.9515 & 4.85 & 0.02 & 0.9793 & 0.9432 & 5.65 & -1.82 \\
\hline & VMD-BC-Elman & 0.9834 & 09565 & 4.35 & -0.14 & 0.9827 & 0.9546 & 4.52 & 0.37 \\
\hline
\end{tabular}


600 Tab.8 As in Tab.6, but for Huaxian station.

\begin{tabular}{cccccccccc}
\hline \multirow{2}{*}{ Types } & \multirow{2}{*}{ Model } & \multicolumn{4}{c}{ Training Dataset } & \multicolumn{4}{c}{ Testing Dataset } \\
\cline { 3 - 9 } Original & SVM & 0.6478 & 0.3580 & 64.08 & 22.47 & 0.5335 & 0.2551 & 74.10 & 19.28 \\
& Elman & 0.7255 & 0.5260 & 47.31 & -1.35 & 0.3596 & -0.3594 & 135.23 & -57.76 \\
\hline \multirow{2}{*}{ EMD-SVM } & 0.8463 & 0.6957 & 30.37 & 8.13 & 0.7819 & 0.5498 & 40.31 & 8.32 \\
& EEMD-SVM & 0.9164 & 0.8352 & 16.45 & 4.26 & 0.8969 & 0.8034 & 19.56 & 3.13 \\
& VMD-SVM & 0.9823 & 0.9555 & 4.44 & 0.45 & 0.9775 & 0.9436 & 5.61 & 0.62 \\
Decomposition & EMD-Elman & 0.9489 & 0.8990 & 10.08 & -3.46 & 0.6860 & 0.4184 & 57.86 & 10.15 \\
& EEMD-Elman & 0.9694 & 0.9393 & 6.06 & 2.47 & 0.8336 & 0.6814 & 31.69 & 10.17 \\
& VMD-Elman & 0.9853 & 0.9629 & 3.70 & 0.01 & 0.9788 & 0.9485 & 5.12 & 0.19 \\
\hline Box-Cox & BC-SVM & 0.8407 & 0.6845 & 31.49 & 13.26 & 0.8013 & 0.5994 & 39.85 & 10.12 \\
transformation & BC-Elman & 0.9648 & 0.9309 & 6.90 & 0.01 & 0.7273 & 0.5202 & 47.73 & 10.17 \\
\hline \multirow{2}{*}{$\begin{array}{c}\text { Decomposition } \\
\text { and Box-Cox }\end{array}$} & VMD-BC-SVM & 0.9835 & 0.9579 & 4.20 & 0.26 & 0.9796 & 0.9473 & 5.24 & 0.24 \\
\hline
\end{tabular}

601 
603 Fig.1 Architectural graph of Elman neural network.

604 Fig.2. Architecture of the proposed VMD-BC-Elman model (CIV= candidate input variables; IV= 605 input variables).

606 Fig.3 The study area showing the runoff observations sites in the Wei River Basin, China.

607 Fig.4 The observed monthly runoff series, the training and testing data sets at three hydrological 608 stations. (a)Zhangjiashan station; (b)Zhuangtou station; (c) Huaxian station.

609 Fig.5 The variability points of monthly runoff time series at three stations. (a)Zhangjiashan station; 610 (b)Zhuangtou station; (c)Huaxian station.

611 Fig.6 Decomposition results by VMD for monthly runoff time series at Zhangjiashan station. (a) IMF1612 IMF4; (b) IMF5-IMF8.

613 Fig.7 Probability density of the candidate input variables of the original runoff time series before and 614 after Box-Cox transformation at Zhangjiashan Station.

615 Fig.8 The correlation coefficients between the output and input variables before and after Box-Cox 616 transformation at Zhangjiashan Station.

617 Fig.9 The flowchart of modeling comparison.

618 Fig.10 The observed $v$ s. predicted runoff in the testing period generated at Zhangjiashan station. (a) 619 Single models; (b) The decomposition-based hybrid models; (c) The Box-Cox transformation-based 620 hybrid models; (d) VMD-BC-SVM and VMD-BC-Elman.

621 Fig.11 Scatter plots of the predicted $v s$. observed runoff in testing period at Zhangjiashan station.

622 Fig.12 Boxplots of the predicted errors generated by 12 models in the testing period at Zhangjiashan 623 station (VMD-BC-Elman, VMD-BC-SVM, BC-Elman, BC-SVM, VMD-Elman, VMD-SVM, VMD624 Elman, EEMD-Elman, EEMD-SVM, EMD-Elman, EMD-SVM are abbreviated as VBE, VBS, BE, 
625 BS, VE, VS, EEE, EES, EE, ES).

626 Fig.13 Taylor diagram depicting the predictive ability of 12 models in the testing period at 627 Zhangjiashan station.

628 Fig.14 The observed vs. predicted runoff in the testing period. (a) Zhuangtou station; (b) Huaxian 629 station. 


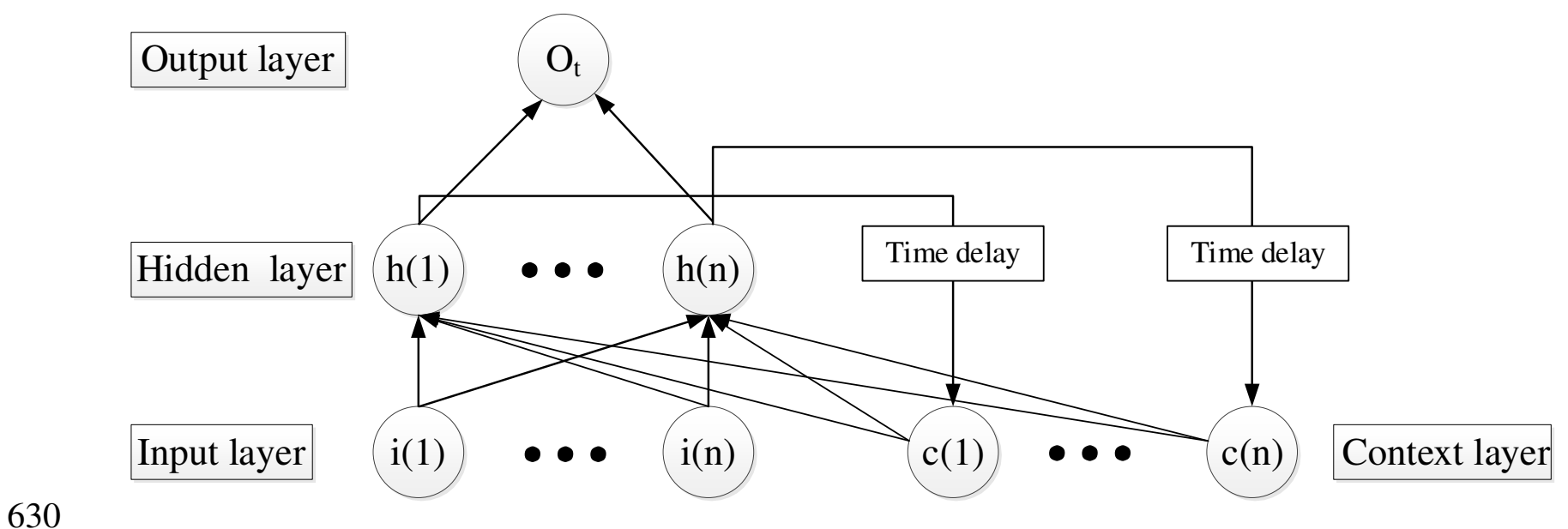

631 Fig.1 Architectural graph of Elman neural network. 


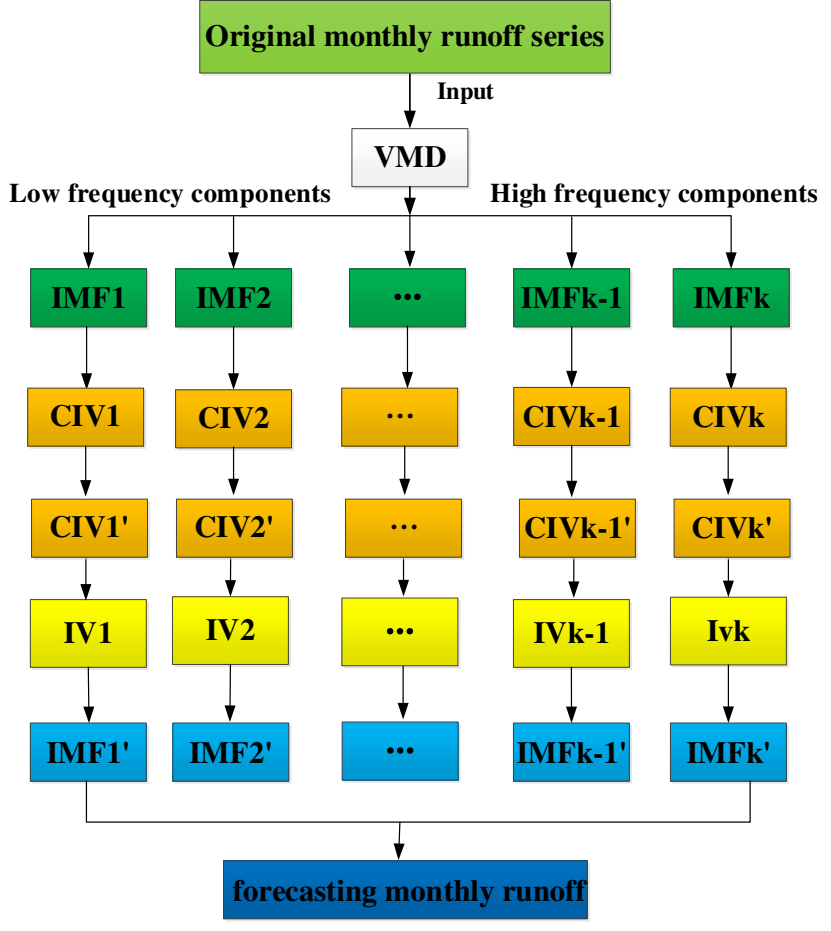

633 Fig.2 Architecture of the proposed VMD-BC-Elman model (CIV= candidate input variables; IV= input 634 variables). 


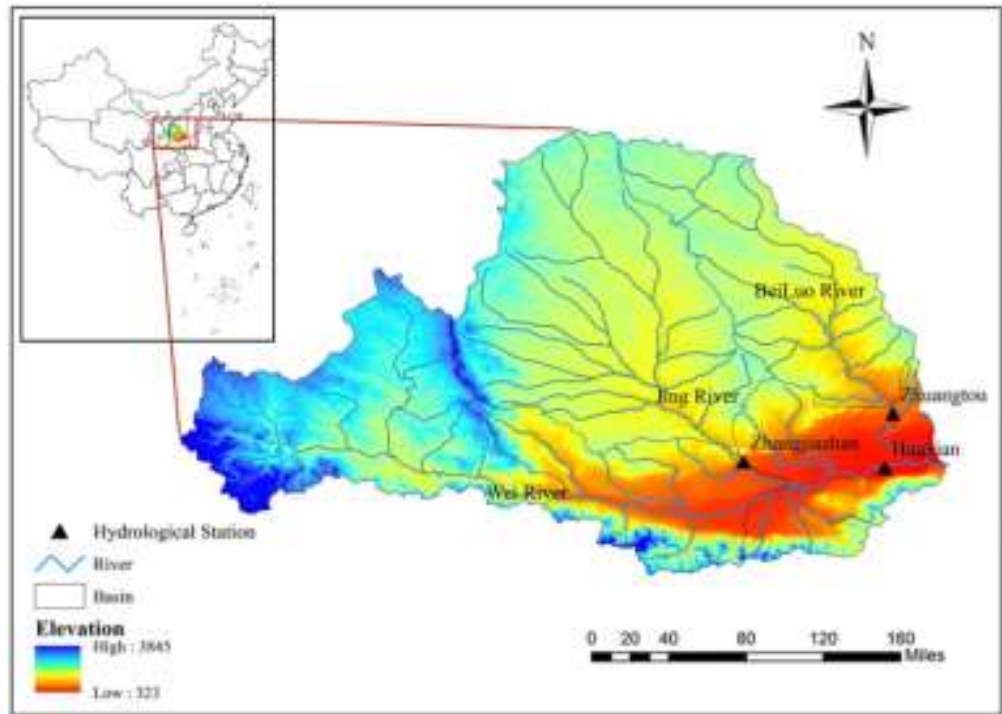

636 Fig.3 The study area showing the runoff observations sites in the Wei River Basin, China. 


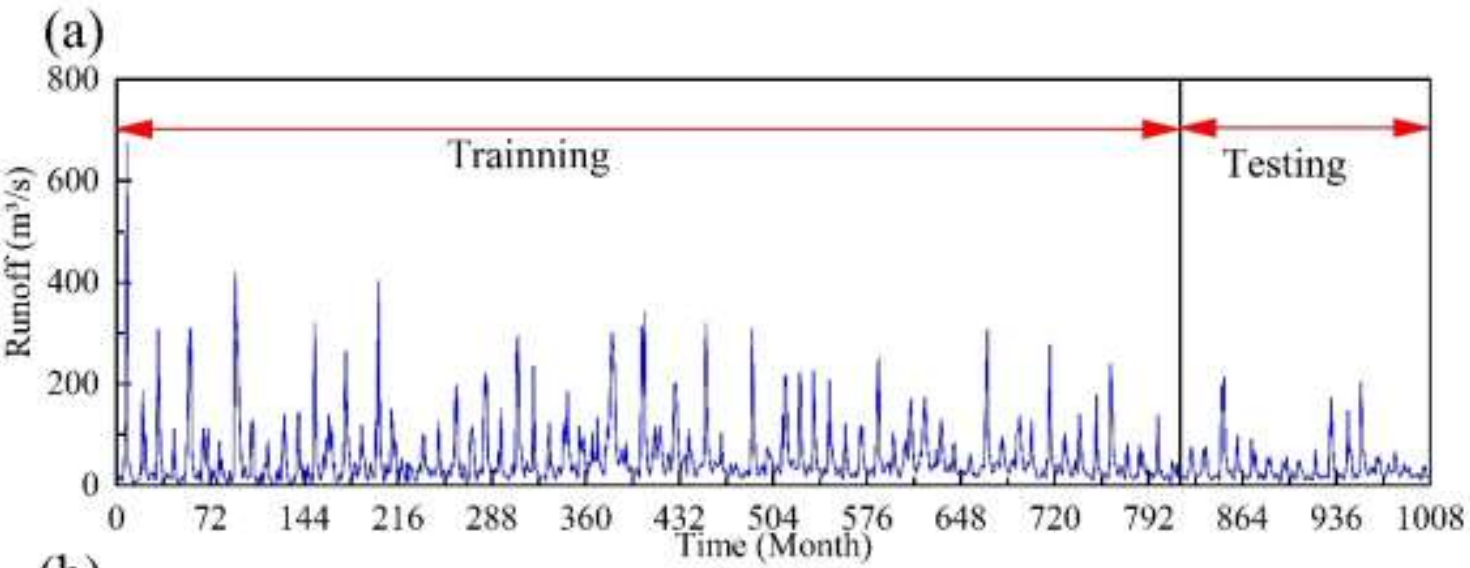

(b)

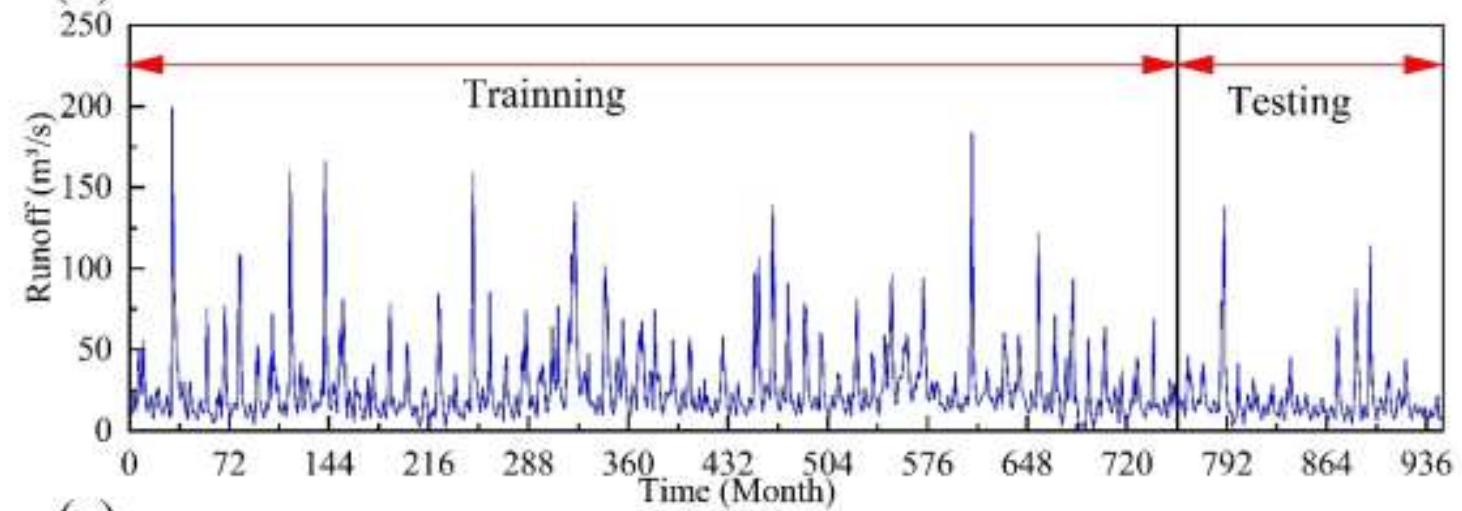

(c)

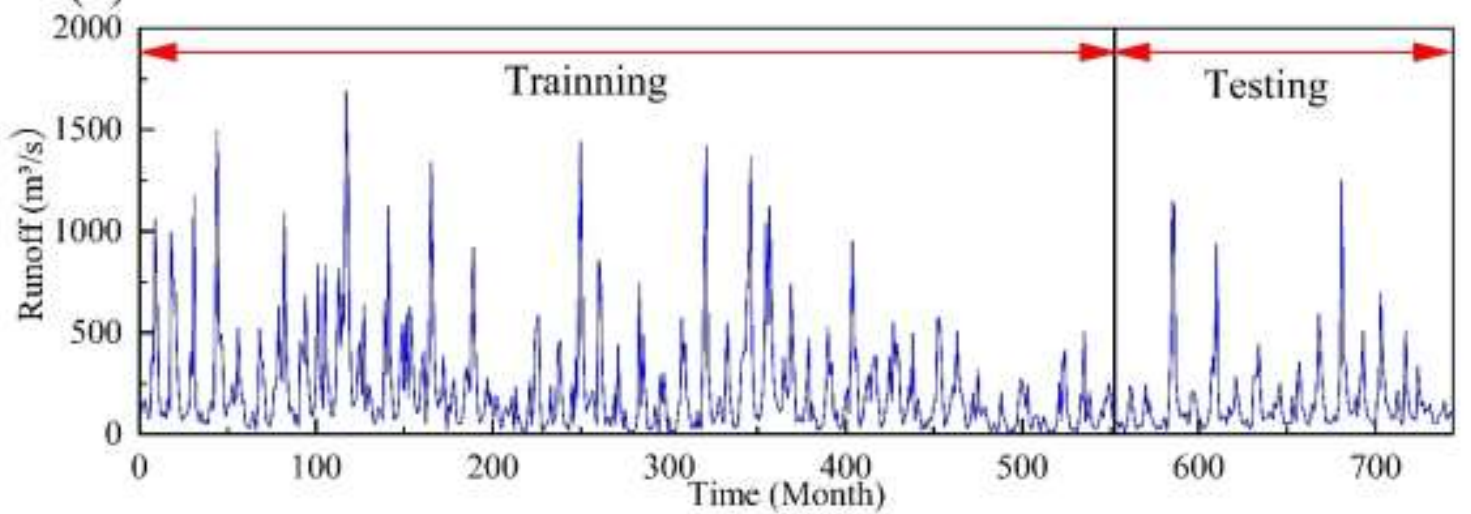

637

638 Fig.4 The observed monthly runoff series, the training and testing data sets at three hydrological

639 stations. (a)Zhangjiashan station; (b)Zhuangtou station; (c) Huaxian station. 

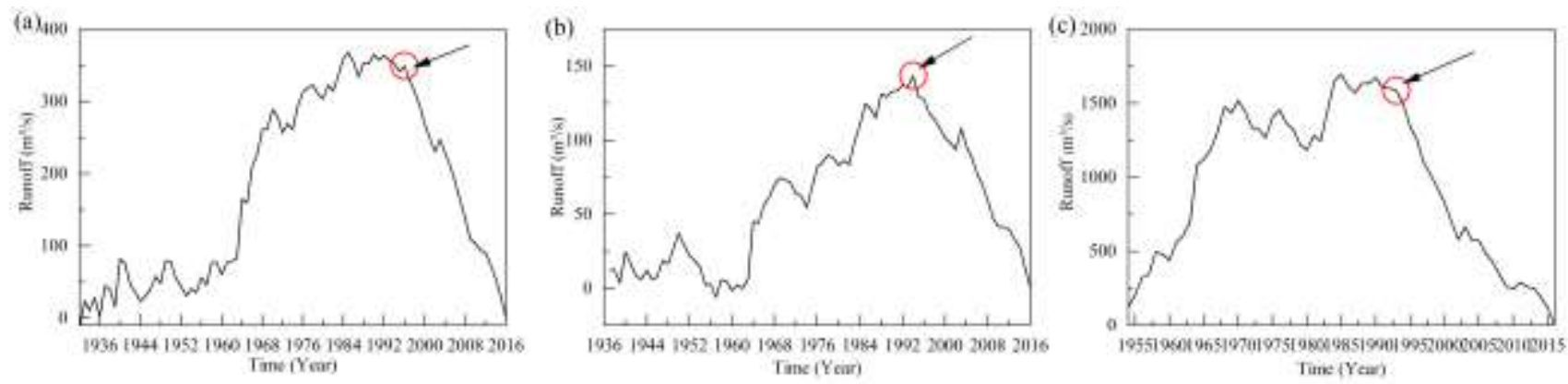

641 Fig.5 The variability points of monthly runoff time series at three stations. (a)Zhangjiashan station; 642 (b)Zhuangtou station; (c)Huaxian station. 

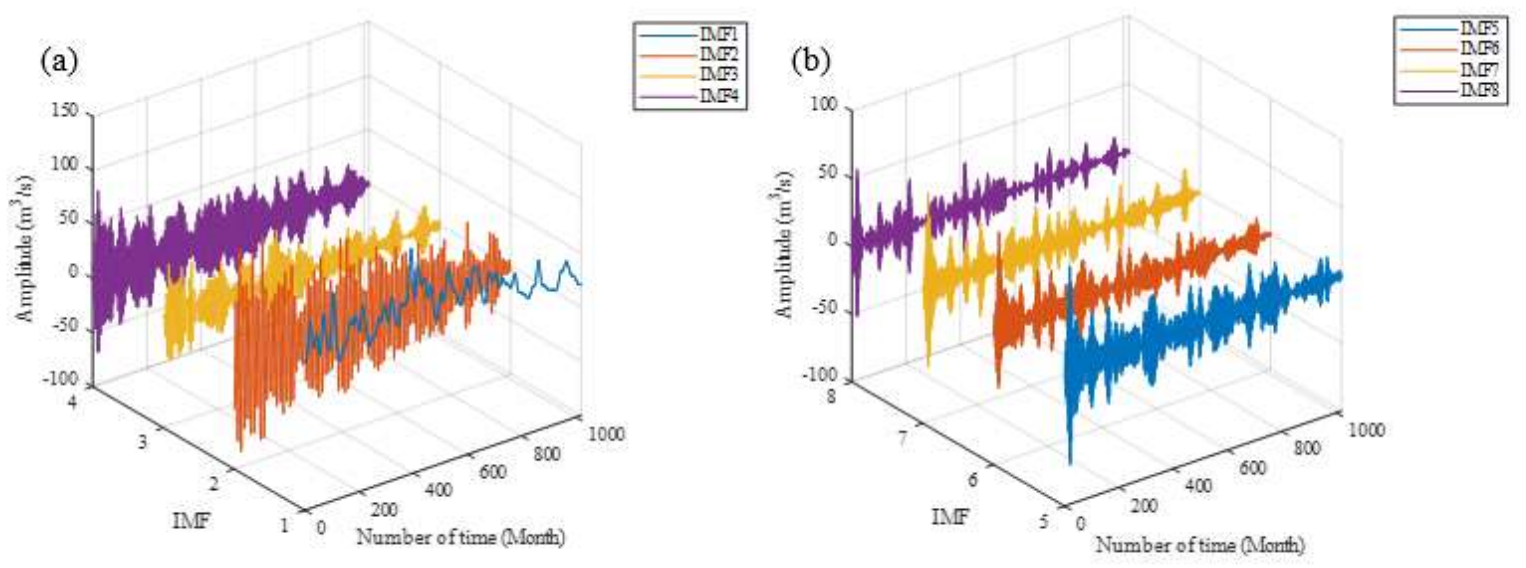

643

644 Fig.6 Decomposition results by VMD for monthly runoff time series at Zhangjiashan station. (a) IMF1645 IMF4; (b) IMF5-IMF8. 
(a) $x_{t-1}$
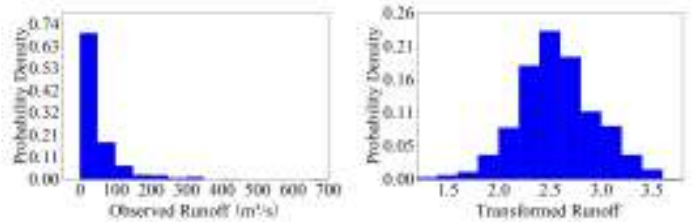

(c) $x_{t-3}$
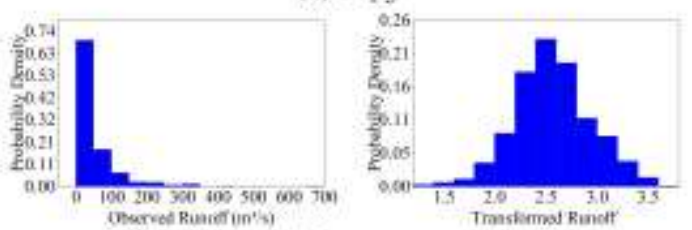

(e) $x_{t-5}$
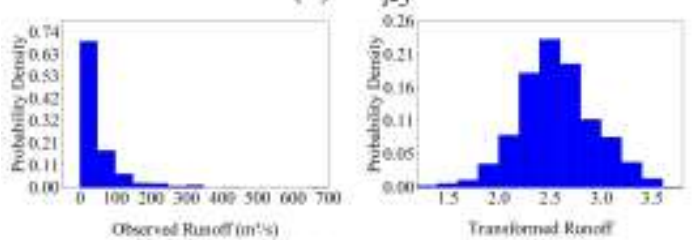

(g) $x$
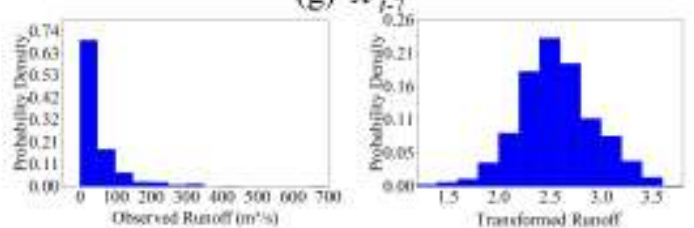

(i) $x_{t, 9}$
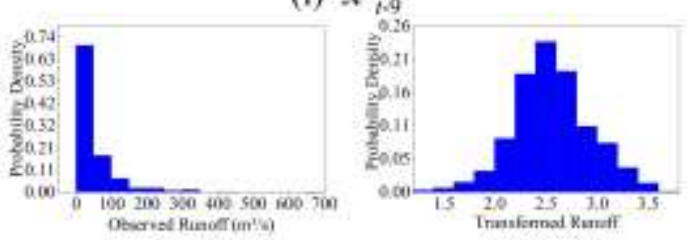

(k) $x_{t-11}$
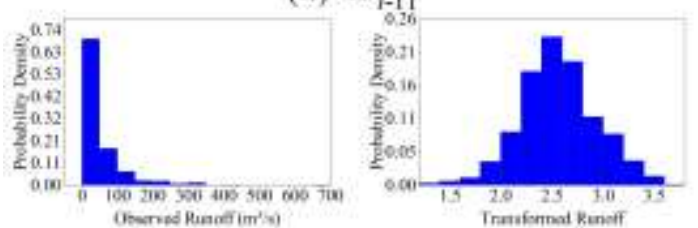

(b) $x_{t-2}$
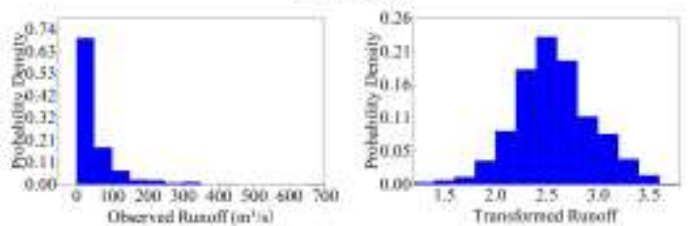

(d) $x_{t-4}$
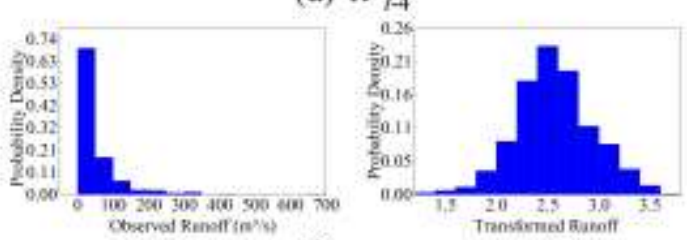

(f) $x$
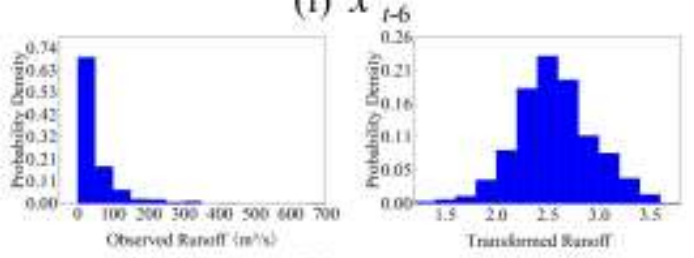

(h) $x$
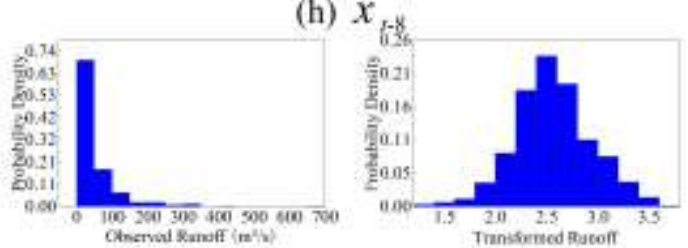

(j) $x_{t-1}$
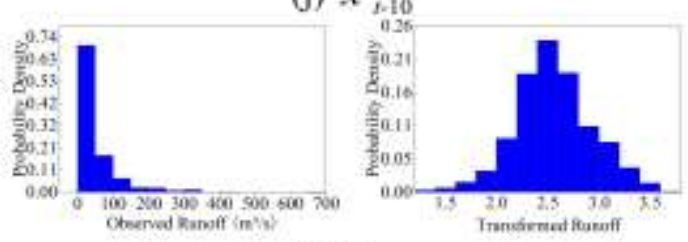

(1) $x$
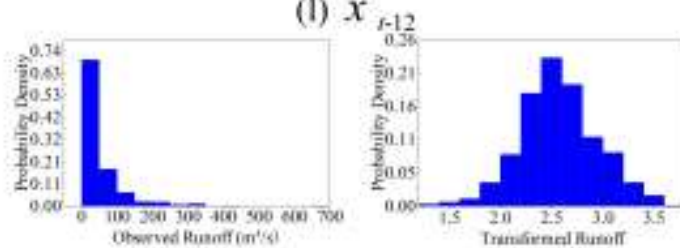

Fig.7 Probability density of the candidate input variables of the original runoff time series before and after Box-Cox transformation at Zhangjiashan Station. 


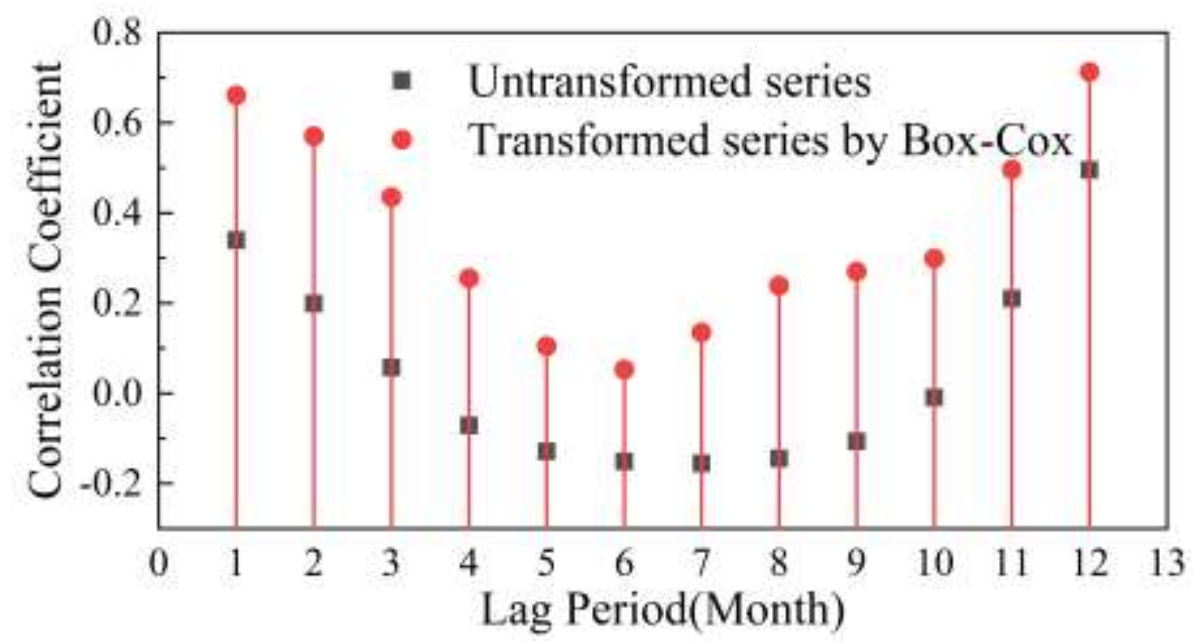

650 Fig.8 The correlation coefficients between the output and input variables before and after Box-Cox 651 transformation at Zhangjiashan Station. 


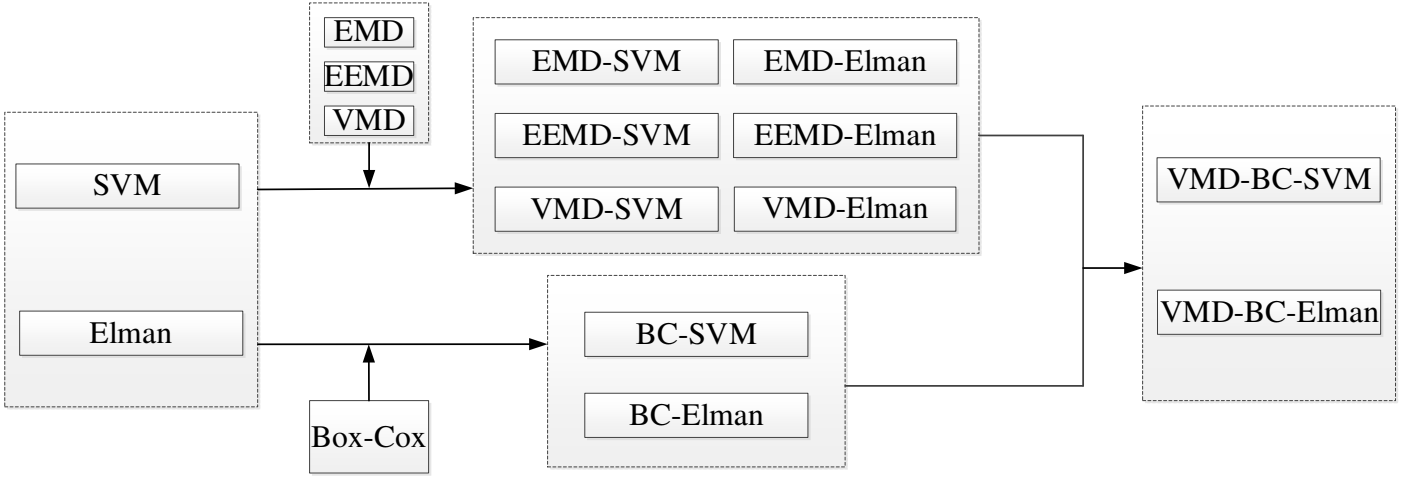

653 Fig.9 The flowchart of modeling comparison. 
(a)
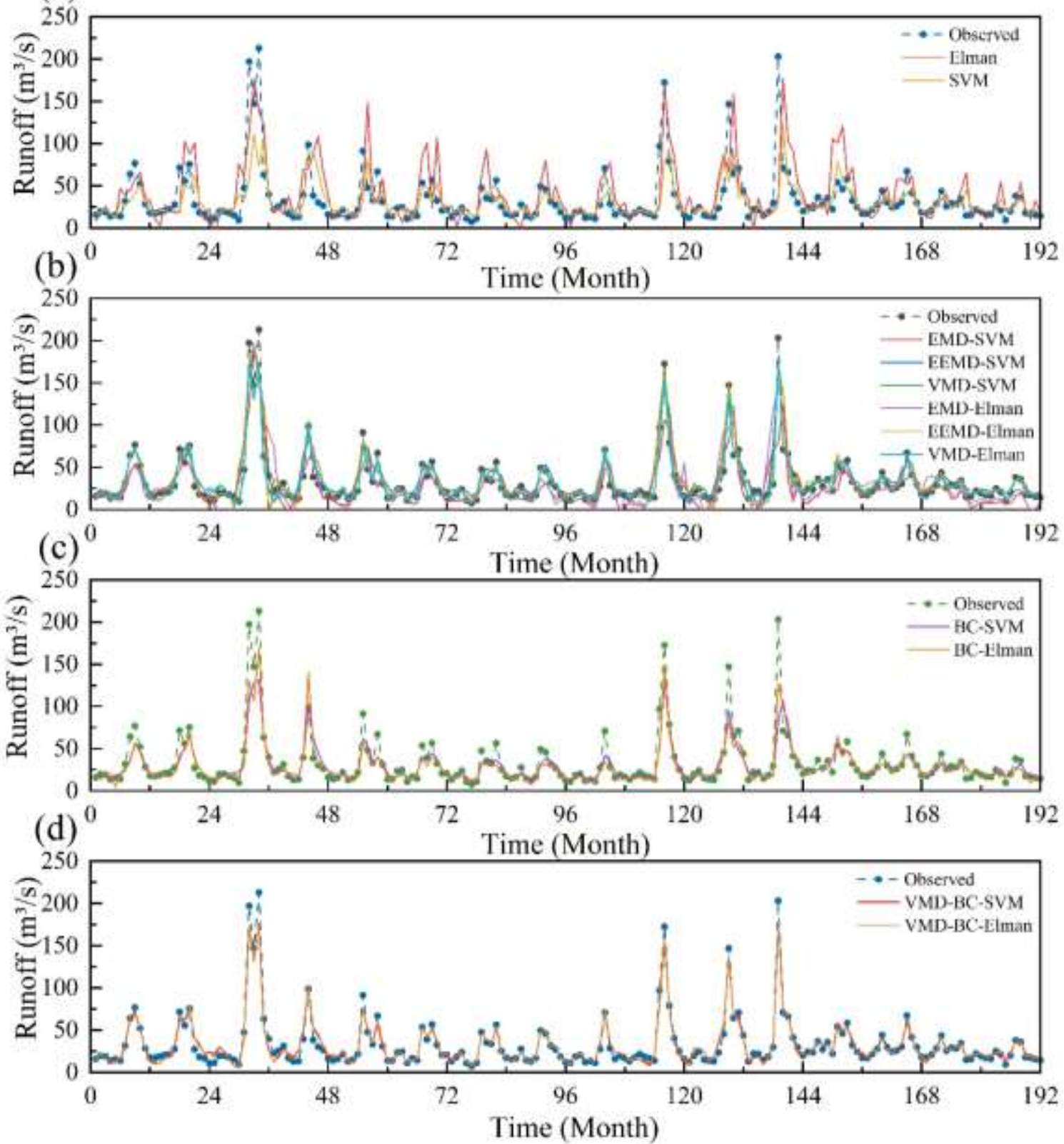

655 Fig.10 The observed $v$ s. predicted runoff in the testing period generated at Zhangjiashan station. (a)

656 Single models; (b) The decomposition-based hybrid models; (c) The Box-Cox transformation-based

657 hybrid models; (d) VMD-BC-SVM and VMD-BC-Elman. 

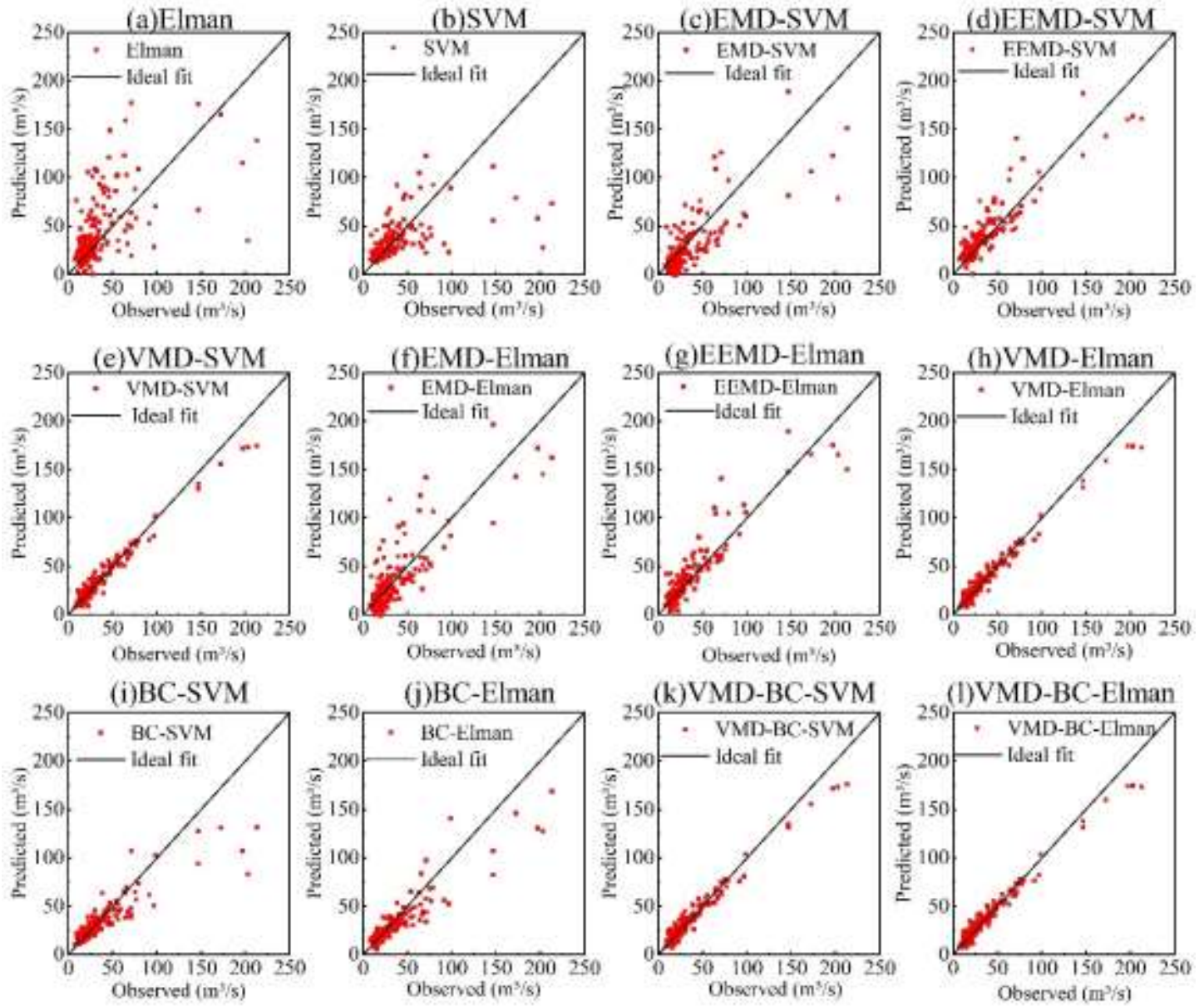

659 Fig.11 Scatter plots of the predicted $v s$. observed runoff in testing period at Zhangjiashan station. 


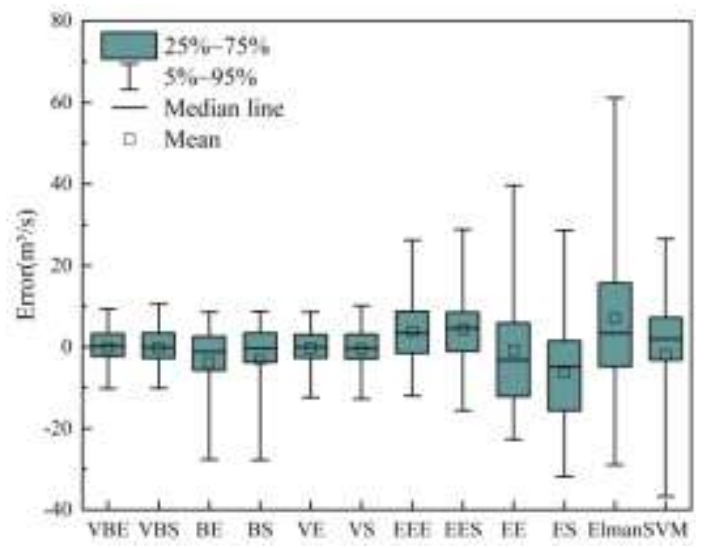

661 Fig.12 Boxplots of the predicted errors in the testing period at Zhangjiashan station (VMD-BC-

662 Elman, VMD-BC-SVM, BC-Elman, BC-SVM, VMD-Elman, VMD-SVM, VMD-Elman, EEMD-

663 Elman, EEMD-SVM, EMD-Elman, EMD-SVM are abbreviated as VBE, VBS, BE, BS, VE, VS, 664 EEE, EES, EE, ES). 


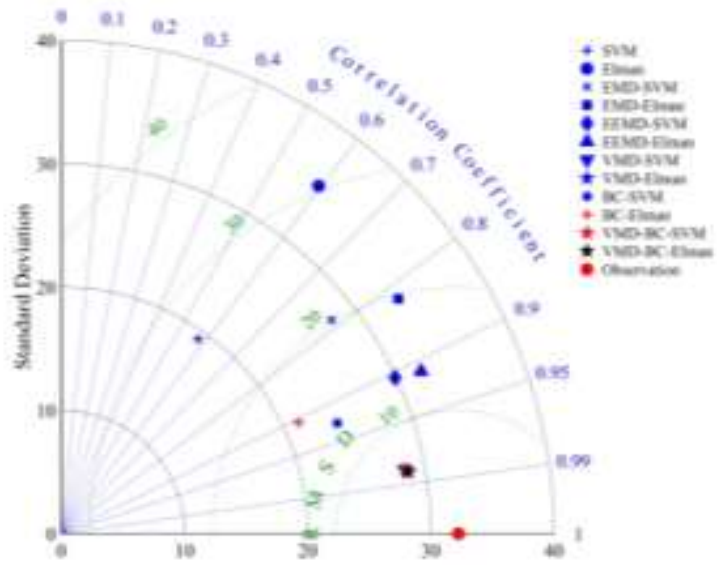

666 Fig.13 Taylor diagram depicting the predictive ability of 12 models in the testing period at 667 Zhangjiashan station. 
(a)
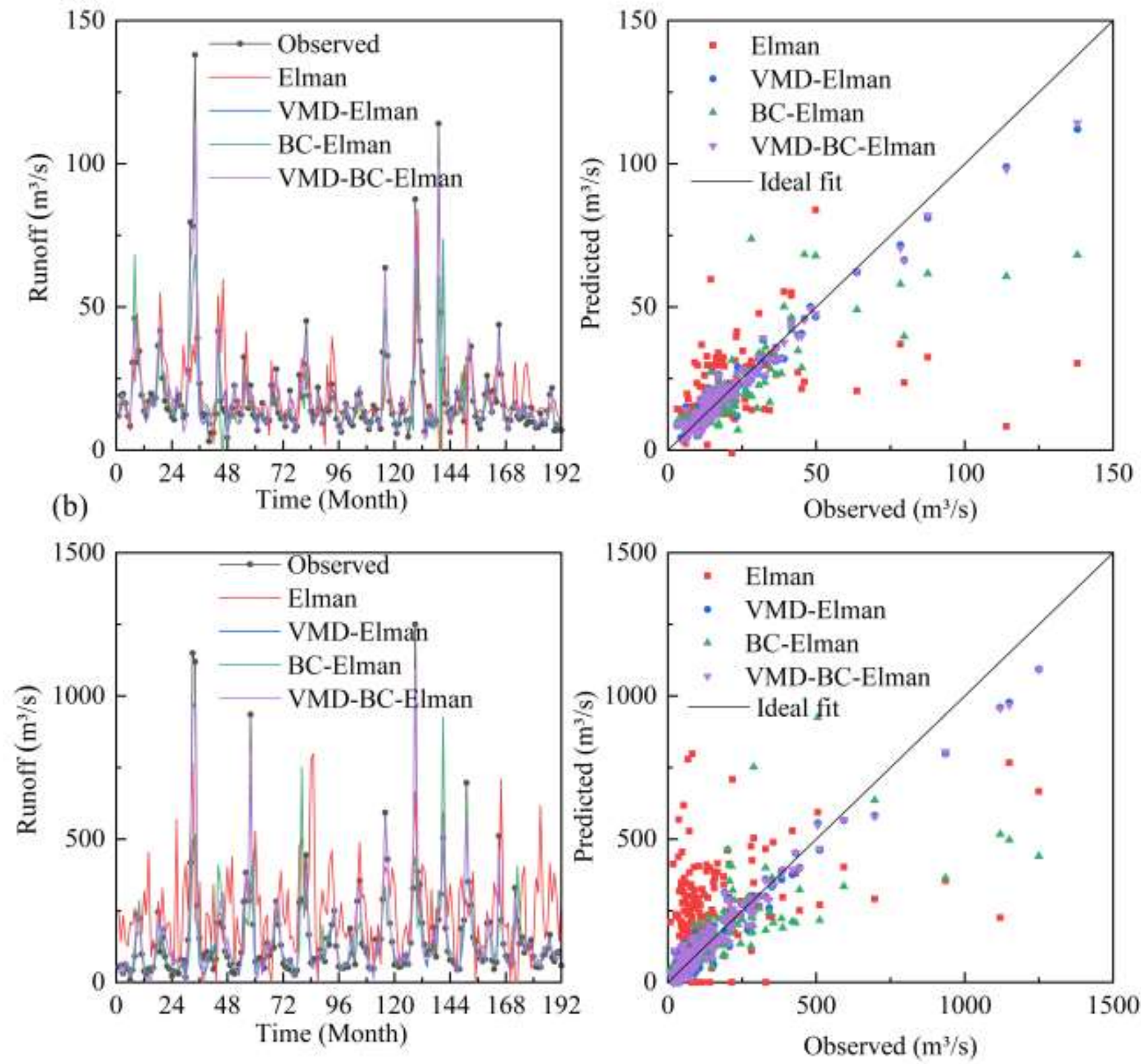

669 Fig.14 The observed $v s$. predicted runoff in the testing period. (a) Zhuangtou station; (b) Huaxian 670 station. 